\title{
On Kontsevich's Hochschild cohomology conjecture
}

\author{
Po Hu, Igor Kriz and Alexander A. Voronov
}

\begin{abstract}
Generalizing a conjecture of Deligne, Kontsevich proposed that there should be a notion of Hochschild cohomology of algebras over the little cube operad (or its chain complex) which in a natural way generalizes Hochschild cohomology of associative algebras. He moreover conjectured that the Hochschild cohomology, in this new sense, of an algebra over the little $k$-cube operad is an algebra over the little $(k+1)$-cube operad. In this paper, we precisely state and prove this conjecture.
\end{abstract}

\section{Introduction}

A conjecture of Deligne stated that the Hochschild cohomology complex of an associative algebra has a natural structure of a 2-algebra, i.e. an algebra over the chain complex version of the 2 -cube operad. This indicated a remarkable connection between the deformation theory of associative algebras, and the geometry of configuration spaces of points in the plane. There are several known proofs of Deligne's conjecture, see [Tam98, Tam03, Vor00, MS01, KS99]. The purpose of the present paper is to prove a generalization conjectured by Kontsevich [Kon99], calling for an analogue of Deligne's conjecture for algebras over the little $k$-cube operad.

The first problem is to define a suitable generalization of the Hochschild cohomology complex. Kontsevich [Kon99] proposes to do this by modifying the Quillen cohomology complex, but that approach forces some restrictions (in fact, it only seems to work for the little $k$-cube operad and, as stated, only in chain complexes over fields of characteristic 0). A key feature of our approach is that we give a completely natural definition of the Hochschild cohomology complex, not restricted to those situations. In fact, all of our constructions in principle work for any operad, and in a closed symmetric monoidal category [Mac71]. Nevertheless, to avoid technical problems, we shall still stick to specific cases. Namely, in the statement of our theorems, we shall assume that $\mathcal{B}$ is the category of sets, or $K$-modules where $K$ is a field (not necessarily of characteristic 0 ). There is at least one substantially different case of interest, namely the case of spectra ( $S$-modules [EKMM97]). However, homotopical algebra in that case is more difficult, and will not be discussed here.

Now consider operads $\mathcal{C}$ in the category of simplicial sets. An example of special interest to us is the operad $\mathcal{C}_{k}$ which is the set of singular simplices of the operad of little $k$-cubes [May72]. In the beginning of the next section, we will introduce the notion of $\mathcal{C}$-algebras $R$ in the category $s \mathcal{B}$, and $(\mathcal{C}, R)$-modules. Furthermore, for $(\mathcal{C}, R)$-modules $M, N$, we will construct a 'derived mapping object'

$$
\operatorname{RHom}_{(\mathcal{C}, R)}(M, N)
$$

in the homotopy category of $s \mathcal{B}$. Then our main result can be stated as follows.

Received 28 March 2004, accepted in final form 8 December 2004.

2000 Mathematics Subject Classification 18D50, 55P48, $13 \mathrm{D} 03$.

Keywords: operads, Hochschild cohomology, algebraic structures.

The authors were supported by the NSF.

This journal is (c) Foundation Compositio Mathematica 2006. 


\section{P. Hu, I. Kriz And A. A. Voronov}

Theorem 1. Let $R$ be a cofibrant $\mathcal{C}_{k}$-algebra (in $s \mathcal{B}$ as above). Then there is a functorial model of

$$
\operatorname{RHom}_{\left(\mathcal{C}_{k}, R\right)}(R, R)
$$

which is a $\mathcal{C}_{k+1}$-algebra.

Tamarkin [Tam00] previously proved a purely algebraic version of Theorem 1 for the Quillen cohomology complex [Qui68] (also known as the deformation complex), working in the category of chain complexes rather than simplicial modules. His proof works over fields of characteristic 0 , and does not include the statement of Deligne's original conjecture (the case of $k=1$ ). It uses the fact that the little cube chain operad in that case is formal and Koszul.

Theorem 1 is based on the geometry of the little $k$-cube operad. Its proof will be completely 'derived' and based on a general principle (cf. [Dun88]) that 'commuting $\mathcal{C}_{k^{-}}$and $\mathcal{C}_{1}$-structures give a $\mathcal{C}_{k+1}$-structure'. In the case of Hochschild cohomology of $R$, the $\mathcal{C}_{k}$-structure is induced by the $\mathcal{C}_{k}$-structure of $R$, the $\mathcal{C}_{1}$-structure from the Yoneda product. Our proof applies to modules over fields of any characteristic (as well as sets), and does include the case $k=1$. In the statement of the theorem, the assumption that $R$ be cofibrant should not be regarded as a restriction, since for non-cofibrant algebras the correct notion of Hochschild cohomology is obtained by first taking a cofibrant replacement: this leads to the right notion of Hochschild cohomology in the case $k=1$.

The present paper is organized as follows. In the next section, we shall reduce Theorem 1 to a much more general context, which may be of independent interest as a generalization of the Kontsevich conjecture. We will introduce the notion of operads fibered over a given operad $\mathcal{C}$ in simplicial sets. Using Boardman-Vogt's $\square$-product of operads [BV73], we will state in Theorem 3 that for a certain class of operads $Q$ fibered over $\mathcal{C}$, which we call special, there is a notion of Hochschild cohomology object constructed from $Q$, and that moreover this object has the structure of a $\mathcal{C} \square \mathcal{C}_{1}$-algebra. We also prove a simplicial version of the theorem of Dunn [Dun88] comparing $\mathcal{C}_{k} \square \mathcal{C}_{\ell}$ with $\mathcal{C}_{k+\ell}$

There are three facts which together will reduce the proof of Theorem 1 to Theorem 3. First, a $\mathcal{C}_{k}$-algebra gives rise to an example of a special operad fibered over $\mathcal{C}_{k}$. Second, the two ensuing notions of Hochschild cohomology coincide. Third, we have $\mathcal{C}_{k} \square \mathcal{C}_{1} \simeq \mathcal{C}_{k+1}$. All of these statements are proven in $\S 2$ with the exception of the 'special' property: that is technical, and left to $\S 6$.

In $\S \S 3$ and 4 , we will describe the technical machinery used to prove Theorem 3 . This technique can be separated into two steps. First, in $\S 3$, we shall consider 'lax algebras' over an operad $\mathcal{C}$ and show how they may be turned into strict algebras over a different, but weakly equivalent, operad $\overline{\mathcal{C}}$. In $\S 4$, we shall introduce an additional, 'vertical' category structure on a lax algebra, which will allow us to get a $\overline{\mathcal{C}} \square \mathcal{C}_{1}$-algebra. Both constructions must be discussed also in categories 'enhanced' over a given category $\mathcal{B}$ (we deliberately choose a different name than 'enriched' which is a different notion, although also related to our considerations). In $\S 5$, we will apply the techniques of $\S \S 3$ and 4 to our main example, which will give the proof of Theorem 3.

\section{Special operads fibered over $\mathcal{C}$}

In this section, we shall reduce Theorem 1 to another statement, which can be phrased in a more general context. However, we begin by filling in the missing definitions in the statement of Theorem 1 .

Recall [Mac71] that a closed symmetric monoidal category is a category $\mathcal{B}$ with a symmetric monoidal structure $\triangle$ and an 'internal Hom' functor Hom : $\mathcal{B}^{O p} \times \mathcal{B} \rightarrow \mathcal{B}$ with a natural bijection

$$
\operatorname{Mor}(A \otimes B, C) \cong \operatorname{Mor}(A, \operatorname{Hom}(B, C)),
$$

satisfying certain axioms. We shall work in the category $s \mathcal{B}$ of simplicial objects in $\mathcal{B}$. 


\section{KOntSEviCH's CONJECTURE}

For a set $S$ and an object (or morphism) $X$ of $\mathcal{B}$, let

$$
S \otimes X=\coprod_{S} X
$$

We say that an object $R$ of $\mathcal{B}$ is a $\mathcal{C}$-algebra for an operad $\mathcal{C}$ in sets if there are structure maps

$$
\mathcal{C}(n) \otimes(R \otimes \cdots \otimes R) \rightarrow R
$$

satisfying the usual axioms (see, e.g., [KM95]). Now an important point is that here (and analogously in other places below), the same definition may be used for an operad in simplicial sets and an object of $s \mathcal{B}$ (this simply means that maps (2) exist on each simplicial level, and are natural with respect to simplicial structure). For a $\mathcal{C}$-algebra $R$, a $(\mathcal{C}, R)$-module is an object $M$ of $\mathcal{B}$ together with structure maps

$$
\mathcal{C}(n) \otimes(M \otimes R \otimes \cdots \otimes R) \rightarrow M
$$

satisfying the usual axioms (cf. [KM95]). If $C_{R}$ is the monad in $s \mathcal{B}$ defining free $(\mathcal{C}, R)$-modules, then define for $(\mathcal{C}, R)$-modules $M, N$,

$$
\operatorname{Hom}_{(\mathcal{C}, R)}(M, N)
$$

as the equalizer of the two obvious maps

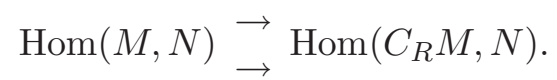

(One is induced by $C_{R} M \rightarrow M$, the other by the natural map $\operatorname{Hom}(M, N) \rightarrow \operatorname{Hom}\left(C_{R} M, C_{R} N\right)$, composed with the map induced by the map $C_{R} N \rightarrow N$.)

We will be considering Quillen (closed) model structures on certain categories, which will be needed for homotopy theory in those categories. A (closed) model structure on a complete cocomplete category consists of three classes of morphisms called fibrations, cofibrations and equivalences. (The word closed may be omitted as it carries no meaning.) All of the information on closed model structures needed in this paper, and all the methods of constructing closed model structures we shall need can be found in [Qui67, DS95]. One should remark that on many categories there are many closed model structures which have the same equivalences and hence lead to the same homotopy theory. However, when doing constructions, one typically needs to fix a closed model structure in order to control the homotopy behavior of the objects produced by the construction.

The most basic case needed is the category of simplicial sets (see [DS95, $\S 11.1]$ ). In this category, there is a closed model structure where cofibrations are injective maps (more precisely sequences of injective maps), and equivalences are maps of simplicial sets which produce homotopy equivalences after simplicial realization. (It then follows that fibrations are so-called Kan fibrations, but that is less important to us.) Next, recall [DS95, $\S 11.2$ ] that a Quillen model structure in $s \mathcal{B}$ is obtained as follows. Let $U$ be the forgetful functor from $s \mathcal{B}$ to simplicial sets. Then a morphism $f$ in $s \mathcal{B}$ is a fibration or equivalence if and only if $U f$ is a fibration (equivalence). We say that fibrations and equivalences are created by the forgetful functor $U$.

Remark. Actually, it turns out that in the two cases we consider in this paper that $f$ is a cofibration if and only if $U f$ is a cofibration also, i.e. cofibrations are also created by $U$ : the case of simplicial sets is tautological, and in the case of simplicial vector spaces that category is an abelian category, equivalent to the category of $\geqslant 0$-graded chain complexes by functors which preserve injections and surjections, and hence every injection or surjection which induces an equivalence splits. In addition, all simplicial surjections are fibrations, but cofibrations are characterized as maps having the left lifting property with respect to fibration equivalences, so cofibrations are also simplicial injections. 


\section{P. Hu, I. Kriz And A. A. Voronov}

There is also a canonical Quillen model structure on the category of $\mathcal{C}$-algebras (with equivalences and fibrations same as in $s \mathcal{B}$, i.e. created by the forgetful functor), and for a cofibrant $\mathcal{C}$-algebra $R$, there is a canonical Quillen model structure on the category of $(\mathcal{C}, R)$-modules (again with equivalences and fibrations same as in $s \mathcal{B}$ ). This is proven by a 'small object argument', and the proof works for categories of algebraic structures in $s \mathcal{B}$ of very general kinds. (The small object argument is described in $[\mathrm{DS} 95, \S 7.12])$. We shall refer to representatives of an $s \mathcal{B}$-equivalence class as models.

With the Quillen model structure established, now recall that an object $X$ is called cofibrant (respectively, fibrant) if the map from the initial object to $X$ (respectively, from $X$ to the terminal object) is a cofibration (respectively, fibration). A cofibrant replacement (respectively, fibrant replacement) of an object $M$ (respectively, $N$ ) is a map

$$
M^{\prime} \rightarrow M
$$

(respectively, $N \rightarrow N^{\prime}$ ) which is a fibration equivalence (respectively, cofibration equivalence) and $M^{\prime}$ (respectively, $N^{\prime}$ ) is cofibrant (respectively, fibrant). We define

$$
\operatorname{RHom}_{(\mathcal{C}, R)}(M, N)
$$

as $\operatorname{Hom}_{(\mathcal{C}, R)}\left(M^{\prime}, N^{\prime}\right)$ where $M^{\prime}$ is a cofibrant replacement of $M$ and $N^{\prime}$ is a fibrant replacement of $N$. In our cases, cofibrant and fibrant replacement can be made functorial, so (4) is well defined. Nevertheless, cofibrant and fibrant replacements are not canonical, and hence it is appropriate to address the question of comparing the different RHom when different selections are made.

To this end, one uses the following technique. Let $I$ be the standard simplicial model of the unit interval. Then we have objects of the form

$$
I \otimes M
$$

which are cylindrical objects (see $[\mathrm{DS} 95, \S 4.1])$ in the sense that the two maps

$$
i_{0}, i_{1}: M \rightarrow I \otimes M
$$

induced by the inclusions of the endpoints to $I$ are equivalences and $i_{0} \coprod i_{1}: M \coprod M \rightarrow I \otimes M$ is a cofibration. We define a homotopy of two maps $f_{0}, f_{1}: M \rightarrow N$ to be a map $I \otimes M \rightarrow N$ which, when composed with $i_{j}$, gives $f_{j}$. This is a particular example of what is known as a Quillen left homotopy, but the present notion has more features which we will find useful. In particular, the functor $I \otimes$ ? has a right adjoint which we will denote by $F(I, ?)$, and also the internal Hom-functor $\operatorname{Hom}_{(\mathcal{C}, R)}$ obeys the relation

$$
\operatorname{Hom}_{(\mathcal{C}, R)}(I \otimes M, N) \cong \operatorname{Hom}_{(\mathcal{C}, R)}(M, F(I, N)) \cong F\left(I, \operatorname{Hom}_{(\mathcal{C}, R)}(M, N)\right) .
$$

To be precise, the $F(I, ?)$ on the right-hand side of $(5)$ is in $s \mathcal{B}$ rather than the category of $(\mathcal{C}, R)$ modules. Note, however, that since $F(I, ?)$ is a limit, the forgetful functor from $(\mathcal{C}, R)$ to $s \mathcal{B}$ preserves $F(I, ?)$, so it is given by the same construction in $s \mathcal{B}$ as in $(\mathcal{C}, R)$-modules. All of this is formal. In addition, it is true in our case that $F(I, N)$ is a co-cylindrical object (satisfying properties dual to cylindrical object; also known as path object, see [DS95, $\S 4.12]$ ) if $N$ is fibrant (since, again, this is true in $s \mathcal{B}$ ). It follows that for any other cofibrant replacement $M^{\prime \prime} \rightarrow M$ there is a comparison map

$$
M^{\prime} \rightarrow M^{\prime \prime}
$$

commuting with the specified maps into $M$ and, moreover, unique up to homotopy (in our sense). Hence, by the same principle, we also obtain a map

$$
M^{\prime \prime} \rightarrow M^{\prime},
$$




\section{Kontsevich's CONJECTURE}

and the compositions are homotopic to the identity. We call this a homotopy equivalence of $(\mathcal{C}, R)$ modules. However, then applying $\operatorname{Hom}_{(\mathcal{C}, R)}\left(?, N^{\prime}\right)$, we obtain a homotopy equivalence in $s \mathcal{B}$, which is an equivalence. The treatment of fibrant replacements is adjoint.

We shall now turn to the reduction of Theorem 1 to another statement.

Definition. Let $S$ be a simplicial set, i.e. a functor $\Delta^{O p} \rightarrow$ Sets. Then $S$ can also be viewed as a category $\bar{S}$ with objects $\coprod_{n} S_{n}$, and morphisms $\phi: s \rightarrow t$ where $\phi \in \operatorname{Mor}\left(\Delta^{O p}\right), s \in S_{n}$ for some $n$, and $\phi(s)=t$. Let $\Gamma$ be any category. Then a $s \Gamma$-object fibered over $S$ is, by definition, a functor

$$
F: \bar{S} \rightarrow \Gamma \text {. }
$$

We shall write $F_{s}=F(s)$ for $s \in S_{n}$. For a map $i: S \rightarrow T$ of simplicial sets, we have a functor $i^{*}$ from $s \Gamma$-objects over $T$ to $s \Gamma$-objects over $S$, given by

$$
i^{*}(F)=F \circ \bar{i} .
$$

We shall also make use of the left adjoint to $i^{*}$, which we shall denote by $i_{\sharp}$.

Note that $s \Gamma$-objects fibered over the constant simplicial set $*$ are precisely $s \Gamma$-objects, which helps justify the terminology.

Specifically, we will now be interested in the case $\Gamma=s \mathcal{B}$ as above. Clearly, for every pair of objects $X, Y$ of $s \mathcal{B}$ fibered over simplicial sets $S, T$, there is a canonical object $X \otimes Y$ fibered over $S \times T$.

Remark. Simplicial realization $|\cdot|: s s \mathcal{B} \rightarrow s \mathcal{B}$ is defined as the diagonal functor [KM95]:

$$
|X|_{n}=X_{n n} .
$$

By definition, we have

$$
|X \otimes Y|=|X| \otimes|Y|
$$

We will sometimes drop the symbol $|\cdot|$ from our notation.

Let $\mathcal{C}$ be an operad in simplicial sets. Then an operad $Q$ in $s \mathcal{B}$ fibered over $\mathcal{C}$ consists of objects $Q(n)$ of $s \mathcal{B}$ fibered over $\mathcal{C}(n)$, with $\Sigma_{n}$-action, and unity for $n=1$ and, for each of the compositions

$$
\gamma: \mathcal{C}(k) \times \mathcal{C}\left(n_{1}\right) \times \cdots \times \mathcal{C}\left(n_{k}\right) \rightarrow \mathcal{C}\left(n_{1}+\cdots+n_{k}\right),
$$

a composition

$$
\gamma_{\sharp}\left(Q(k) \otimes Q\left(n_{1}\right) \otimes \cdots \otimes Q\left(n_{k}\right)\right) \rightarrow Q\left(n_{1}+\cdots+n_{k}\right)
$$

satisfying the obvious axioms analogous to the operad axioms [May72].

Now simplicial sets fibered over a simplicial set $S$ are precisely simplicial sets $X$ over $S$, i.e. arrows $X \rightarrow S$. A morphism in this category is a fibration, cofibration of equivalence if and only if it has the corresponding property in simplicial sets. If $U_{S}$ is the forgetful functor from objects and morphisms of $s \mathcal{B}$ fibered over $S$ to simplicial sets fibered over $S$, then we say that a morphism $f$ in $s \mathcal{B}$ fibered over $S$ is a fibration or equivalence if and only if $U_{S} f$ is a fibration or equivalence. By [DS95], again, this defines a Quillen model structure on the category of objects of $s \mathcal{B}$ fibered over $S$.

Finally, on operads (similarly as any type of algebraic structure) in objects and morphisms of $s \mathcal{B}$ fibered over $\mathcal{C}$ we consider the closed model structure taking as fibrations (respectively, equivalences) sequences of maps $(A(n) \rightarrow \mathcal{C}(n))_{n}$ which are fibrations (equivalences) in the category of objects and morphisms of $s \mathcal{B}$ fibered over $\mathcal{C}(n)$.

Definition. For any simplicial set $S$, let $h=h_{S}: S \rightarrow *$ be the collapse map, and let

$$
Z=B\left(\mathcal{C}(\ell), \mathcal{C}(1)^{\times \ell}, \mathcal{C}(0)^{\times \ell}\right) .
$$




\section{P. Hu, I. Kriz And A. A. Voronov}

We shall call an operad $Q$ fibered over $\mathcal{C}$ special if, for every $\ell$, the map

$$
B\left(Q(\ell), Q(1)^{\otimes \ell}, Q(0)^{\bigotimes \ell}\right) \rightarrow h_{Z}^{*} Q(0)
$$

(where $B$ is the two-sided bar construction), induced from the composition map

$$
h_{Z \sharp} B\left(Q(\ell), Q(1)^{\otimes \ell}, Q(0)^{\otimes \ell}\right) \rightarrow Q(0)
$$

is an equivalence.

Remark. If $Q$ is fibrant in the category of $s \mathcal{B}$-operads fibered over $\mathcal{C}$ such that $\mathcal{C}(0)=*, \mathcal{C}(1) \simeq *$, and the unit inclusion

$$
i:\{*\} \rightarrow \mathcal{C}(1)
$$

is a cofibration equivalence (which we are assuming), then the counit map

$$
i^{*} Q(1)=h_{\sharp} i_{\sharp} i^{*} Q(1) \rightarrow h_{\sharp} Q(1)
$$

is an equivalence. We note that (8) is a map of monoids in $s \mathcal{B}$. We shall denote

$$
Q_{1}=i^{*} Q(1) .
$$

Thus, for fibrant operads $Q$ over $\mathcal{C}$, we can replace $Q(1)$ by $Q_{1}$ in (7). Of course, every operad in $s \mathcal{B}$ over $\mathcal{C}$ can be replaced by a fibrant model. We shall make use of this below.

Now for a monoid $R$ in $s \mathcal{B}$, a module over $R$ is an object $M$ of $s \mathcal{B}$ with a map

$$
R \otimes M \rightarrow M
$$

satisfying the usual axioms. Clearly, $R$-modules are precisely algebras over a monad $C_{R}$ of the form

$$
C_{R}(X)=R \otimes X
$$

Thus, we have a canonical closed model structure on $R$-modules for any monoid $R$. As before, we define

$$
\operatorname{Hom}_{R}(M, N)
$$

as the equalizer of the two obvious maps

$$
\operatorname{Hom}(M, N) \underset{\operatorname{Hom}}{\rightarrow}\left(C_{R} M, N\right),
$$

and define $\operatorname{RHom}_{R}(M, N)=\operatorname{Hom}_{R}\left(M^{\prime}, N^{\prime}\right)$ where $M^{\prime}$ is a cofibrant replacement of $M$ and $N^{\prime}$ is a fibrant replacement of $N$ (with derived independence on the choice of $M^{\prime}$ and $N^{\prime}$, for $s \mathcal{B}$ as above). Note that an example of a $Q_{1}$-module in the preceding remark is $Q(0)$.

Now for two operads $\mathcal{C}, \mathcal{D}$ in simplicial sets, following [BV73], define an operad $\mathcal{C} \square \mathcal{D}$ as the quotient of the free operad $\mathcal{F}$ on $\mathcal{C} \amalg \mathcal{D}=(\mathcal{C}(n) \amalg \mathcal{D}(n))_{n}$ modulo identifying the $\mathcal{F}$-operad operations on objects of $\mathcal{C}, \mathcal{D}$ with the corresponding operations in $\mathcal{C}, \mathcal{D}$, (this includes units), and the following key relation: for $\alpha \in \mathcal{C}(m), \beta \in \mathcal{D}(n)$,

$$
\alpha(\underbrace{\beta, \ldots, \beta}_{m \text { times }})=\beta(\underbrace{\alpha, \ldots, \alpha}_{n \text { times }}) \sigma
$$

where $\sigma$ is a certain permutation reordering terms. To describe this permutation, consider the 'row-by-row' lexicographical bijection

$$
\rho_{1}:\{1, \ldots, m\} \times\{1, \ldots, n\} \rightarrow\{1, \ldots, m n\}
$$

(i.e. $(11 \mapsto 1,12 \mapsto 2, \ldots, 1 n \mapsto n, 21 \mapsto n+1, \ldots)$ ), and the 'column-by-column' lexicographical bijection

$$
\rho_{2}:\{1, \ldots, m\} \times\{1, \ldots, n\} \rightarrow\{1, \ldots, m n\}
$$




\section{KOntSEviCH's CONJECTURE}

(i.e. $(11 \mapsto 1,21 \mapsto 2, \ldots, m 1 \mapsto m, 12 \mapsto m+1, \ldots))$. The point is that on the left-hand side of (9), the entries are ordered 'row by row' whereas on the right-hand side they are ordered 'column by column'. Since permutations on operads act on the right, we conclude that

$$
\sigma=\rho_{2} \circ\left(\rho_{1}\right)^{-1} .
$$

Proposition 2. There is a canonical map of operads

$$
\phi: \mathcal{C}_{k} \square \mathcal{C}_{\ell} \rightarrow \mathcal{C}_{k+\ell},
$$

which, in the $n$th term

$$
\phi:\left(\mathcal{C}_{k} \square \mathcal{C}_{\ell}\right)(n) \rightarrow \mathcal{C}_{k+\ell}(n),
$$

is a $\Sigma_{n}$-equivariant homotopy equivalence $\left(\Sigma_{n}\right.$ denotes the symmetric group on $n$ elements). An analogous result also holds if we work in the category of topological spaces (rather than simplicial sets).

Remark. Note that the proposition implies that both in the topological and simplicial cases, the map $\phi$ induces an equivalence of monads in the sense of [May72].

We shall prove this at the end of this section. The second statement of the proposition is a theorem of Dunn [Dun88, Theorem 2.9]. We give a proof of the first statement, which we need for technical reasons, at the end of this section. To this end, to fix notation, we will also need to briefly recall the main ideas of Dunn's argument. The simplicial extension of Dunn's result contains no substantially new idea, but due to subtleties must be handled with care. We will now restate Theorem 1 as follows.

Theorem 3. Let $Q$ be a special cofibrant fibrant operad in $s \mathcal{B}$ fibered over $\mathcal{C}$ where $s \mathcal{B}$ is as above and $\mathcal{C}$ is an operad in simplicial sets with $\mathcal{C}(0)=*, \mathcal{C}(1) \simeq *$, and such that the $\Sigma_{n}$-action on $\left(\mathcal{C} \square \mathcal{C}_{1}\right)(n)$ is free. Then there is a model of

$$
\operatorname{RHom}_{Q_{1}}(Q(0), Q(0))
$$

which has a natural structure of a $\mathcal{C} \square \mathcal{C}_{1}$-algebra.

A discussion is needed to see how Theorem 3 implies Theorem 1. Recall that, in general, for a monad $C$, a $C$-functor $D$ (i.e. a functor with a structure map $D C \rightarrow D$ satisfying the usual axioms) and a $C$-algebra $X$, we can define

$$
D \otimes_{C} X
$$

as the coequalizer of the two maps

$$
D C X \underset{\rightarrow}{\rightarrow} D X
$$

given by the structure maps $D C \rightarrow D, C X \rightarrow X$. Now for an operad $\mathcal{C}$ in the category of simplicial sets, $\mathcal{C}$-algebras are algebras of the monad

$$
C X=\coprod_{n \geqslant 0} \mathcal{C}(n) \otimes \Sigma_{n} X^{\otimes n}
$$

We shall define $C$-functors $D_{\ell}$ as follows:

$$
D_{\ell} X=\coprod_{n \geqslant 0} \mathcal{C}(n+\ell) \otimes_{\Sigma_{n}} X^{\otimes n} .
$$

The following statement is obvious upon a moment's reflection. 


\section{P. Hu, I. Kriz And A. A. Voronov}

Proposition 4. Let $\mathcal{C}$ be an operad in simplicial sets and assume in addition $\mathcal{C}(0)=*$ (the one-point simplicial set). Let $R$ be a $\mathcal{C}$-algebra. Then the object

$$
A(n)=D_{n} \otimes_{C} R
$$

has the natural structure of an operad in $s \mathcal{B}$ fibered over $\mathcal{C}$.

Proof. Recall that because the functor ? $\otimes X$ in $\mathcal{B}$ has a right adjoint, $\otimes$ is distributive under $\amalg$. Now we need to construct structure maps

$$
\begin{aligned}
& \mathcal{C}(n+\ell) \otimes_{\Sigma_{n}} R^{\bigotimes n} \otimes \\
& \left(\mathcal{C}\left(n_{1}+m_{1}\right) \otimes_{\Sigma_{n_{1}}} R^{\bigotimes n_{1}}\right) \otimes \cdots \otimes\left(\mathcal{C}\left(n_{\ell}+m_{\ell}\right) \otimes_{\Sigma_{n_{\ell}}} R^{\bigotimes n_{\ell}}\right) \\
& \left(\mathcal{C}\left(n+n_{1}+\cdots+n_{\ell}+m_{1}+\cdots+m_{\ell}\right) \otimes_{\Sigma_{n_{1}+\cdots+n_{\ell}}} R^{\bigotimes n+n_{1}+\cdots+n_{\ell}}\right)
\end{aligned}
$$

compatible with $\otimes_{C}$. However, realizing that $\otimes$ is nothing but coproducts over various sets, distributivity also applies to this case, and we see that the map (13) is obtained just by applying operad composition in $\mathcal{C}$, and grouping the $\otimes$-powers of $R$. Compatibility with $\otimes_{C}$ is obvious from operad axioms. In addition, the fibered structure is obtained by taking, for an element

$$
x \in \mathcal{C}(n)_{i}
$$

( $i$ denotes the simplicial index) $A(n)_{x}$ to be the coproduct of all

$$
\{y\} \otimes_{\Sigma_{\ell}} R^{\nabla \ell}
$$

where $y \in \mathcal{C}(n+\ell)$ is such that

$$
\gamma(y, 1, \ldots, 1, *, \ldots, *)=x
$$

where $\gamma$ is operad multiplication, and in (14), there are $n 1$ s and $\ell * \mathrm{~s}$.

The reduction from Theorem 1 to Theorem 3 then follows from the following two results.

Lemma 5. The category of $(\mathcal{C}, R)$-modules is equivalent to the category of $h_{\sharp} A(1)$-modules. This equivalence of categories carries $R$ to $A(0)$. Moreover, if $R$ is a cofibrant $\mathcal{C}$-algebra (with $s \mathcal{B}$ as above), this is a Quillen equivalence, i.e. passes on to an equivalence of Quillen homotopy categories.

Proof. The equivalence of categories is established by the fact that both categories consist of algebras over the same monad:

$$
X \mapsto h_{\sharp} A(1) \otimes X .
$$

Indeed, it suffices to consider the case when $R$ is a free $\mathcal{C}$-algebra, i.e. $R=C X$. Then

$$
\begin{aligned}
A(1) & =D_{1} \otimes_{C} R=D_{1} X \\
& =\coprod_{n \geqslant 0} \mathcal{C}(n+1) \otimes_{\Sigma_{n}} X^{\otimes n} .
\end{aligned}
$$

Now the free $(\mathcal{C}, R)$-module on $M$ is

$$
\coprod_{n \geqslant 0} \mathcal{C}(n+1) \otimes_{\Sigma_{n}}\left(X^{\otimes n} \otimes M\right)=A(1) \otimes M .
$$

(By abuse of notation, we treat $h_{\sharp}$ as the forgetful functor, so this is the same as (15).)

Now if $R$ is a cofibrant $\mathcal{C}$-algebra, both Quillen model structures on the respective categories are defined in the same way. 


\section{Kontsevich's CONJECTURE}

Remark. Note that this is analogous to a method used by Zhu [Zhu96] for vertex operator algebras (see also [DLM98]). Concretely, vertex operator algebras are close to the notion of algebra over a certain modification to the little 2-disk operad $\mathcal{D}$ (see [Hua97]). One could elaborate a lot more on that, but in the rough analogy, the notion of module over a vertex operator algebra $V$ corresponds to the notion of $(\mathcal{D}, V)$-module. Now Zhu [Zhu96] describes an associative algebra $A$ with the property that, for a rational vertex operator algebra $V$, irreducible $V$-modules $M$ are in bijective correspondence with irreducible $A$-modules. However, the algebra $A$ is not a precise analogue of $h_{\sharp} A(1)$ : in [Zhu96], the algebra $A$ is finite-dimensional and only acts on the top weight part of $M$.

Theorem 6. Let $\mathcal{C}=\mathcal{C}_{k}$ be the little cube operad, and let $R$ be a free $\mathcal{C}_{k}$-algebra. Let $A$ be defined as above in Proposition 4. Then $A$ is special (although not fibrant).

We shall prove this theorem in $\S 6$.

Remark. There are other examples of special operads. For example, working in spaces (one can get to simplicial sets by applying the singular set functor), let, for $e \in \mathcal{C}_{k}(n)$, and a based CW complex $X$,

$$
\left(\Phi_{k}(X)\right)_{e}=\operatorname{Map}((e, \partial e),(X, *)) .
$$

Then there is a standard way to put a topology on

$$
\Phi_{k}(X)(n)=\bigcup_{e \in \mathcal{C}_{k}(n)} \Phi_{k}(X)_{e},
$$

making $\Phi_{k}(X)$ an operad fibered over the topological version of $\mathcal{C}_{k}$. It can be shown that $\Phi_{k}(X)$ is special if $X$ is $(k-1)$-connected.

On the other hand, it is easy to construct operads $\mathcal{C}$ in simplicial sets such that, for $R=C X$ the associated operad $A$ fibered over $\mathcal{C}$ is not special: it suffices to take a free operad on a set (in the category of operads $\mathcal{C}$ with unit and $\mathcal{C}(0)=*$ ).

We shall conclude this section with a proof.

Proof of Proposition 2. We shall first prove the statement for the category of topological spaces, reviewing essentially the ideas of Dunn [Dun88]. In this case, let $\mathcal{C}_{k}$ denote the original topological space models of the little cube operads rather than the singular set model. In this setting, the map (10) is obtained by sending a configuration of $n$ little cubes

$$
\left(c_{1}, \ldots, c_{n}\right) \in \mathcal{C}_{k}(n)
$$

to

$$
\left(c_{1} \times I^{\ell}, \ldots, c_{n} \times I^{\ell}\right) \in \mathcal{C}_{k+\ell}(n),
$$

and a configuration of $n$ little cubes

$$
\left(d_{1}, \ldots, d_{n}\right) \in \mathcal{C}_{\ell}(n)
$$

to

$$
\left(I^{k} \times d_{1}, \ldots, I^{k} \times d_{n}\right) .
$$

We will not construct a homotopy inverse of (10) on the whole $\mathcal{C}_{k+\ell}(n)$, but instead on a certain subspace $\mathcal{C}_{k+\ell}(n)^{\prime}$ which is weakly equivalent. To define this subspace, put $m=k+\ell$. We shall call an $n$-tuple of little cubes

$$
e=\left(e_{1}, \ldots, e_{n}\right)
$$

which is an element of $\mathcal{C}_{m}(n)$ small if the following condition is satisfied: there exists a $p$-tuple of little cubes

$$
f=\left(f_{1}, \ldots, f_{p}\right)
$$




\section{P. Hu, I. Kriz And A. A. Voronov}

forming an element of $\mathcal{C}_{k}(p)$ and a $q$-tuple of little cubes

$$
g=\left(g_{1}, \ldots, g_{q}\right)
$$

forming an element of $\mathcal{C}_{\ell}(q)$ such that every little cube $e_{i}$ lies in the interior of precisely one set

$$
f_{h} \times g_{j},
$$

and every set (17) contains at most one little cube $e_{i}$. The space $\mathcal{C}_{m}(n)^{\prime}$ is the subspace of $\mathcal{C}_{m}(n)$ consisting of precisely all small $n$-tuples. Now we claim that the inclusion $\mathcal{C}_{m}(n)^{\prime} \subset \mathcal{C}_{m}(n)$ is a homotopy equivalence. Indeed, let $e$ be as in (16). Then, for $\lambda \in(0,1]$, define $\lambda e$ as the little cube configuration obtained by scaling each little cube $e_{i}$ by a factor $\lambda$ in its center. Then we know that $\lambda e \in \mathcal{C}_{m}(n)$, and if $e$ is an element of $\mathcal{C}_{m}(n)^{\prime}$, then so is $\lambda e$. Furthermore, it is easy to see that for every $e$ there exists a $\lambda$ such that $\lambda e \in \mathcal{C}_{m}(n)^{\prime}$ (the statement is true trivially if every little cube is replaced by one point, namely its center). By the Lebesgue number theorem, for every compact subset $K \subset \mathcal{C}_{m}(n)$, there exists a $\lambda \in(0,1]$ such that $\lambda K \subset \mathcal{C}_{m}(n)^{\prime}$. Furthermore, t.Id, $t \in[\lambda, 1]$ is a homotopy between $K$ and $\lambda K$, which moreover stays in $\mathcal{C}_{m}(n)^{\prime}$ if $K \subset \mathcal{C}_{m}(n)^{\prime}$. Clearly, this construction is $\Sigma_{n}$-equivariant. By the Whitehead theorem, the inclusion $\mathcal{C}_{m}(n)^{\prime} \subset \mathcal{C}_{m}(n)$ is a $\Sigma_{n}$-weak equivalence and, hence, in fact a $\Sigma_{n}$-homotopy equivalence, since $\mathcal{C}_{m}(n)$ is a $\Sigma_{n}$-CW complex.

Now we will construct a right inverse $\psi$ to the map $\phi$ when restricted to $\mathcal{C}_{m}(n)^{\prime}$. In fact, this construction is obvious: simply compose $f$ with $p$ copies of $g$, and each entry with either $*$ or the appropriate elements of $\mathcal{C}_{k}(1), \mathcal{C}_{\ell}(1)$ to ensure that $\phi \psi=I d_{\mathcal{C}_{m}(n)^{\prime}}$. It should be noted that the map is well defined and continuous, because its value on an element $e$ does not depend on the choice of $f, g$ : any two choices have a 'common subdivision', which produces the same element by the fundamental relation (9). To be more precise, if $A, B$ are two sets of disjoint little cubes in $I^{k}$, the common subdivision of $A, B$ is

$$
\{a \cap b \mid a \in A, b \in B\} .
$$

(While ordering of the cubes of course matters in the operad structure, we do not have to specify it in this definition, as any two orderings are related by the symmetric group action and, hence, any ordering will do.)

Note that we are not yet done: we must still produce a homotopy left inverse to $\phi$. However, now let

$$
\left(\mathcal{C}_{k} \square \mathcal{C}_{\ell}\right)(n)^{\prime}=\phi^{-1}\left(\mathcal{C}_{m}(n)^{\prime}\right) .
$$

First of all, note that the inclusion

$$
\left(\mathcal{C}_{k} \square \mathcal{C}_{\ell}\right)(n)^{\prime} \subset\left(\mathcal{C}_{k} \square \mathcal{C}_{\ell}\right)(n)
$$

is a weak equivalence by the same argument as above: we may emulate the homotopy corresponding to multiplying $e$ by $t$ by composing an element of $\left(\mathcal{C}_{k} \square \mathcal{C}_{\ell}\right)(n)$ with $n$ copies of $\gamma\left(t .1_{k}, t .1_{\ell}\right)$ where $\gamma$ is operad composition, and $1_{k} \in \mathcal{C}_{k}(1), 1_{\ell} \in \mathcal{C}_{\ell}(1)$ are the unit elements. So we are done if we can show that $\psi \phi=I d$ on $\left(\mathcal{C}_{k} \square \mathcal{C}_{\ell}\right)(n)^{\prime}$. However, this is just a refinement of the above argument that $\psi$ did not depend on the choice of $f, g$ : one may form common subdivisions with the $\mathcal{C}_{k}$ and $\mathcal{C}_{\ell}$ elements $u_{i}$ figuring in the definition of an element of $\left(\mathcal{C}_{k} \square \mathcal{C}_{\ell}\right)(n)^{\prime}$, and use the relation (9) to show that the common subdivision produces the same element as using either $f, g$, or $u_{i}$. Again, everything is $\Sigma_{n}$-equivariant. This concludes the proof of our statement for the category of topological spaces.

We shall now study what changes when we work in the category of simplicial sets, using the singular sets of $\mathcal{C}_{k}(n)$ etc. instead of the actual spaces. In this part of the proof, we will find it convenient to display the simplicial set functor $S$ explicitly, to prevent confusion. Much of the idea is the same. For example, the construction of the map $\phi$ is obtained simply by applying 


\section{KOntSEviCH's CONJECTURE}

the singular set functor to the space level $\phi$ (it is useful to note that there is always a canonical map $S \mathcal{C} \square S \mathcal{D} \rightarrow S(\mathcal{C} \square \mathcal{D})$ ). However, when constructing the map $\psi$, we must adapt the definition of $\mathcal{C}_{m}(n)^{\prime}$. In fact, we must introduce the notion of small singular simplex in $S \mathcal{C}_{m}(n)$ as follows: if we represent the singular simplex by an $n$-tuple of singular simplices

$$
\left(e_{1}, \ldots, e_{n}\right)
$$

in the space of little cube (i.e. for $t \in \Delta_{N}$, for some $N, e_{i}(t)$ is a little cube), then there must exist a uniform (i.e. independent of $t$ ) choice of $f, g$ such that $e_{i}(t)$ satisfy the above condition in place of $e_{i}$ for each $t$. This means, roughly, that the value of a small singular simplex at each $t$ is required to be small, but also the values of the singular simplex must vary only by a 'small' amount. We take $S \mathcal{C}_{m}(n)^{\prime}$ as the simplicial set of small singular simplices in $S \mathcal{C}_{m}(n)$. Then the map $\psi$ may be define completely analogously as in the case of spaces, by passing to singular sets.

It is, further, correct to think of $\psi$ as a right homotopy inverse to $\phi$, as it can be shown that $S \mathcal{C}_{m}(n)^{\prime} \subset S \mathcal{C}_{m}(n)$ is an equivalence: this is a special case of a general theorem stating that for any open covering $\left(U_{i}\right)$ of a space $X$, the inclusion of the sub-simplicial set of the singular set of $X$ consisting of singular simplices whose images are in one of the $U_{i}$ is an equivalence. (In our case, $X$ is the topological $S \mathcal{C}_{m}(n)^{\prime}$.)

However, we must still find a left homotopy inverse to $\phi$. By barycentric subdivision of a simplicial set $T$ we shall mean the simplicial set which is the classifying space of the category whose objects are non-degenerate elements of $T$ and morphisms are faces. Denote the $j$-fold barycentric subdivision of a simplicial set $T$ by $T^{(j)}$. It is a well-known fact that if, for the moment, |?| denotes topological simplicial realization, then there is a canonical homeomorphism

$$
|T| \cong\left|T^{(j)}\right|
$$

On the other hand, it is a standard fact (used, for example, in proving Eilenberg-Steenrod axioms for singular homology) that we have a canonical simplicial map

$$
\iota_{j}: S^{(j)} X \rightarrow S X
$$

for any space $X$, sending the 'algebraic barycenter' to the 'topological barycenter' (obviously, it suffices to consider $j=1$ ). Now the simplicial operad $S \mathcal{C}_{k} \square S \mathcal{C}_{\ell}$ is not (at least a priori) the singular set of any space, although, as pointed out above, there is a canonical map

$$
S \mathcal{C}_{k} \square S \mathcal{C}_{\ell} \rightarrow S\left(\mathcal{C}_{k} \square \mathcal{C}_{\ell}\right)
$$

However, we claim that there nevertheless is a canonical simplicial map

$$
\tilde{\iota}_{j}:\left(S \mathcal{C}_{k} \square S \mathcal{C}_{\ell}\right)^{(j)} \rightarrow S \mathcal{C}_{k} \square S \mathcal{C}_{\ell}
$$

constructed in the same way as the map $\iota_{j}$ for singular sets: the point is that any word in singular simplices making up an element of $\left(S \mathcal{C}_{k} \square S \mathcal{C}_{\ell}\right)_{n}$ can be written (and any relation among such elements remains valid) on restrictions of each of those singular simplices to the same simplex of an (iterated) barycentric subdivision of the standard simplex. We further see that by the same argument as for spaces, upon applying simplicial realization, (19) becomes homotopic to the canonical homeomorphism between the realization of a simplicial set and its iterated barycentric subdivision. Hence, (19) is an equivalence.

Thus, we would be done if we could show that $\phi \circ \tilde{\iota}_{j}$ applied to the $n$th space of the source operads lands in $S \mathcal{C}_{m}(n)^{\prime}$ for some $j$. While this is obviously too much to expect, it is, however, true that for any finite simplicial subset $T$ of $S \mathcal{C}_{k} \square S \mathcal{C}_{\ell}$ there exists a $j$ such that the simplicial subset $T^{(j)}$ of

$$
\left(S \mathcal{C}_{k} \square S \mathcal{C}_{\ell}\right)^{(j)}
$$




\section{P. Hu, I. KRIZ And A. A. Voronov}

consisting of $j$-fold barycentric subdivisions of simplices of $T$ does have the property that

$$
\phi \circ \tilde{\iota}_{j}\left(T^{(j)}\right) \subset\left(S \mathcal{C}_{m}\right)(n)^{\prime}
$$

By the Whitehead theorem, this is enough.

\section{Lax algebras}

This story is complex enough that it seems worth telling for the category of (simplicial) sets first. We use essentially the ideas of [May72, KM95], but as far as we know, they have not been recorded with the specific nuances needed here.

Consider the category $\Sigma$ whose objects are finite sets $\{1, \ldots, n\}, n \geqslant 0$, and morphisms are permutations. Then an operad is, in particular, a functor on $\Sigma$.

Now for any operad $\mathcal{C}$, a lax $\mathcal{C}$-algebra is a category $\Gamma$ where each $n$-ary operation $\mu$ of $\mathcal{C}$ corresponds to a functor

$$
\underline{\mu}: \Gamma^{\times n} \rightarrow \Gamma
$$

Further, for each $\mu \in \mathcal{C}(n), \mu_{i} \in \mathcal{C}\left(n_{i}\right), i=1, \ldots, n$, we are required to have natural coherence morphisms

$$
\phi: \underline{\mu}\left(\underline{\mu}_{1}, \ldots, \underline{\mu}_{n}\right) \rightarrow \underline{\gamma\left(\mu, \mu_{1}, \ldots, \mu_{n}\right)}, \quad \phi: \underline{1} \rightarrow 1
$$

where $\gamma$ is the composition in $\mathcal{C}$, and also for $\kappa:\{1, \ldots, n\} \rightarrow\{1, \ldots, n\} \in \operatorname{Mor}(\Sigma)$,

$$
\phi: \underline{\kappa \mu} \cong \underline{\mu} \kappa^{*}
$$

where $\kappa^{*}: \Gamma^{\times m} \rightarrow \Gamma^{\times n}$ is the categorically induced map by $\kappa$. In addition, the coherence isomorphisms (21), (22) are required to satisfy coherence diagrams expressing a cocycle condition for the isomorphisms (21). This means that for a three-fold composition, applying the coherence isos in either order gives the same result

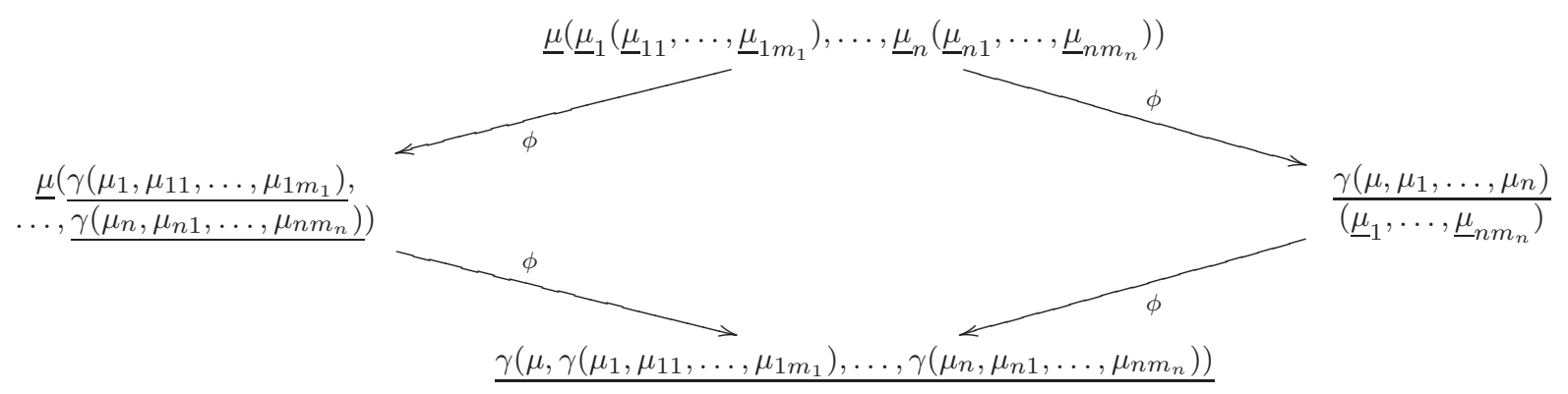

The diagrams coming from operad unit axioms are the following.

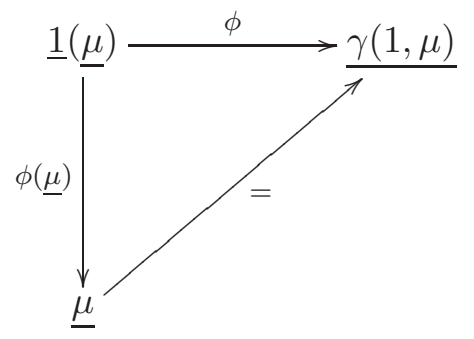




\section{KONTSEVICH's CONJECTURE}

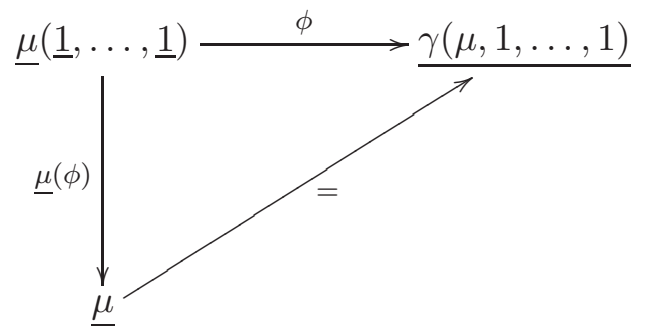

Regarding equivariance, there are two diagrams (associativity and unit) coming from the axiom that $\mathcal{C}: \Sigma \rightarrow$ Sets is a functor. If $\kappa:\{1, \ldots, n\} \rightarrow\{1, \ldots, n\}, \lambda:\{1, \ldots, n\} \rightarrow\{1, \ldots, n\}$ and $\iota=I d:\{1, \ldots, n\} \rightarrow\{1, \ldots, n\}$, the diagrams are as follows.
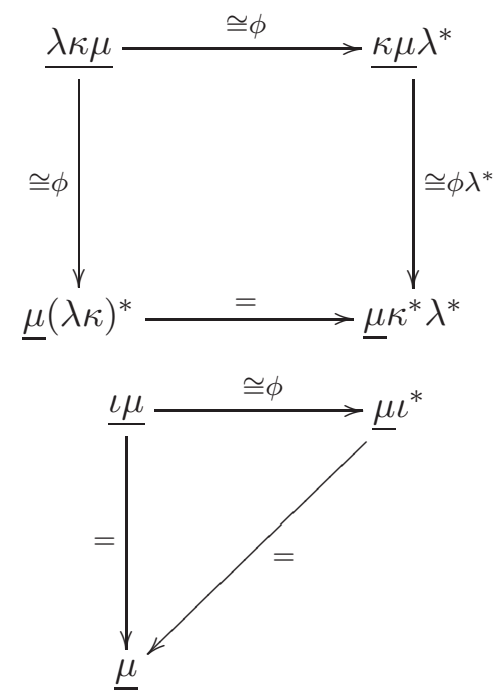

Finally, one diagram comes from the commutation relation between composition and equivariance in an operad. Let $\lambda:\{1, \ldots, n\} \rightarrow\{1, \ldots, n\}, \kappa_{i}:\left\{1, \ldots, k_{i}\right\} \rightarrow\left\{1, \ldots, k_{i}\right\}, i=1, \ldots, n$. Then there is a permutation

$$
\lambda \curlywedge\left(\kappa_{1}, \ldots, \kappa_{n}\right):\left\{1, \ldots, \sum_{i=1}^{n} k_{i}\right\} \rightarrow\left\{1, \ldots, \sum_{i=1}^{n} k_{i}\right\}
$$

given by

$$
\left(\lambda \curlywedge\left(\kappa_{1}, \ldots, \kappa_{n}\right)\right)\left(\sum_{i=1}^{j-1} k_{\lambda(i)}+p\right)=\sum_{i=1}^{\lambda(j)-1} k_{i}+\kappa_{\lambda(j)}(p)
$$

for $j=1, \ldots, n, p=1, \ldots, k_{\lambda(j)}$. The diagram then reads

$$
\begin{aligned}
& \frac{\left(\lambda 乙\left(\kappa_{1}, \ldots, \kappa_{m}\right)\right) \gamma\left(\mu, \mu_{\lambda(1)}, \ldots, \mu_{\lambda(n)}\right)}{\uparrow=} \stackrel{\phi}{\longrightarrow} \frac{\gamma\left(\mu, \mu_{\lambda(1)}, \ldots, \mu_{\lambda(n)}\right)}{\phi\left(\lambda 2\left(\kappa_{1}, \ldots, \kappa_{m}\right)\right)^{*} \uparrow}\left(\lambda \succ\left(\kappa_{1}, \ldots, \kappa_{m}\right)\right)^{*} \\
& \underline{\gamma\left(\lambda \mu, \kappa_{1} \mu_{1}, \ldots, \kappa_{m} \mu_{m}\right)} \quad \underline{\mu}\left(\underline{\mu}_{\lambda(1)}, \ldots, \underline{\mu}_{\lambda(n)}\right)\left(\lambda \succ\left(\kappa_{1}, \ldots, \kappa_{m}\right)\right)^{*} \\
& \uparrow \phi \quad=\uparrow \\
& \underline{\lambda \mu}\left(\underline{\kappa_{1} \mu_{1}}, \ldots, \underline{\kappa_{m} \mu_{m}}\right) \longrightarrow \underline{\mu}^{*}\left(\underline{\mu}_{1} \kappa_{1}^{*}, \ldots, \underline{\mu}_{m} \kappa_{m}^{*}\right)
\end{aligned}
$$

In another formulation, we may consider the free operad $O p(\mathcal{C})$ on $\mathcal{C}$ (i.e. the operad obtained by iterating the operations in $\mathcal{C}$, and performing substitutions and insertions of unit), and assign a 


\section{P. Hu, I. Kriz And A. A. Voronov}

canonical iso to any two elements in $O p(\mathcal{C})(n)$ whose images in $\mathcal{C}(n)$ coincide: these isos are required to be transitive and compatible with substitution and composition. Note that these isos, together with their iterations, now make each $O p(\mathcal{C})(n)$ into a category $G(n)$, whose fiber over each object of $\mathcal{C}$ has a final object. Then $O p(\mathcal{C})$ defines a 2-monad in the sense of Blackwell et al. [BKP89]. Lax $\mathcal{C}$-algebras can then be identified with 2 -algebras over the 2 -monad $O p(\mathcal{C})$.

Now let $\mathcal{C}$ be an operad. We define a simplicial operad $\overline{\mathcal{C}}$ as

$$
\begin{aligned}
\bar{C}(n) & =B G(n)=B_{\operatorname{Obj}(G(n))}(\operatorname{Obj}(G(n)), \operatorname{Mor}(G(n)), \operatorname{Obj}(G(n))) \\
& =B_{O p(C)(n)}(O p(C)(n), \operatorname{Mor}(G(n)), O p(C)(n)) .
\end{aligned}
$$

We have a canonical projection

$$
|\overline{\mathcal{C}}| \rightarrow \mathcal{C}
$$

which is an equivalence since the fiber of $G(n)$ over every element in $\mathcal{C}$ has a final object.

Proposition 7. Let $\Gamma$ be a lax $\mathcal{C}$-algebra. Then $B \Gamma$ is canonically a $\overline{\mathcal{C}}$-algebra.

Proof. Let

$$
A=\left(\begin{array}{ccc}
a_{11} & \ldots & a_{1 n} \\
\vdots & \vdots & \vdots \\
a_{m 1} & \cdots & a_{m n}
\end{array}\right)
$$

be a matrix of morphisms in $\Gamma, T a_{i j}=S a_{i, j+1}$. ( $S, T$ mean source and target.) Let $\phi=\left(\phi_{1}, \ldots, \phi_{n}\right)$ be a composable $n$-tuple in $G(m)$,

$$
\phi_{i}: \sigma_{i-1} \rightarrow \sigma_{i}
$$

Then

$$
\phi A=\left(\phi_{1} \sigma_{0}\left(\begin{array}{c}
a_{11} \\
\vdots \\
a_{m 1}
\end{array}\right), \phi_{2} \sigma_{1}\left(\begin{array}{c}
a_{12} \\
\vdots \\
a_{m 2}
\end{array}\right), \ldots, \phi_{n} \sigma_{n-1}\left(\begin{array}{c}
a_{1 n} \\
\vdots \\
a_{m n}
\end{array}\right)\right) .
$$

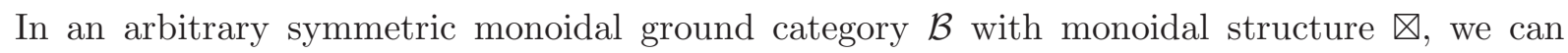
analogously speak of $\mathcal{C}$-algebra for an operad $\mathcal{C}$ (see also Ginzburg and Kapranov [GK94]).

Specifically, we introduce the following terminology: a category $C$ enhanced in $\mathcal{B}$ consists of objects

$$
\operatorname{Obj}(C), \operatorname{Mor}(C) \in \operatorname{Obj}(\mathcal{B})
$$

and morphisms $S, T, I d, \gamma \in \operatorname{Mor}(\mathcal{B}), S, T: \operatorname{Mor}(C) \rightarrow \operatorname{Obj}(C), I d: \operatorname{Obj}(C) \rightarrow \operatorname{Mor}(C), \gamma:$ $\operatorname{Mor}(C) \prod_{\mathrm{Obj}(C)} \operatorname{Mor}(C) \rightarrow \operatorname{Mor}(C)$ (the source of $\gamma$ denotes a pullback), with the usual axioms.

Remark. Note that this differs from a more usual notion of $\mathcal{B}$-enriched category $\Gamma$ in which case we have a set of objects, and for objects $X, Y \in \operatorname{Obj}(\Gamma)$, an object $\underline{\operatorname{Mor}}_{\Gamma}(X, Y) \in \operatorname{Obj\mathcal {B}}$, with morphisms

$$
\underline{\operatorname{Mor}}_{\Gamma}(X, Y) \otimes \underline{\operatorname{Mor}} \Gamma(Y, Z) \rightarrow \underline{\operatorname{Mor}}_{\Gamma}(X, Z) .
$$

Hence, the different terminology. Enriched categories, however, will also be relevant in the next section.

We then have an object

$$
B C \in \operatorname{Obj}(s \mathcal{B})
$$

defined by

$$
B C_{k}=\underbrace{\operatorname{Mor}(C) \prod_{\operatorname{Obj}(C)} \cdots \prod_{\operatorname{Obj}(C)} \operatorname{Mor}(C)}_{k \text { times }}
$$




\section{Kontsevich's CONJECTURE}

Now let $\mathcal{C}$ be an operad in (simplicial) sets. There is a notion of $\mathcal{C}$-algebra $X$ enhanced over $\mathcal{B}$. The structure maps are of the form

$$
\gamma: \mathcal{C}(n) \otimes(\underbrace{X \otimes \cdots \otimes X}_{n \text { times }}) \rightarrow X
$$

with the usual diagrams mimicking the diagrams defining a $\mathcal{C}$-algebra.

Now a lax $\mathcal{C}$-algebra enhanced over $\mathcal{B}$ is a category $C$ enhanced over $\mathcal{B}$ together with functorial structure maps (23) for $X=\operatorname{Obj}(C), \operatorname{Mor}(C)$ and coherence morphism structure of the following form:

For $x \in \mathcal{C}(n), y_{i} \in \mathcal{C}\left(k_{i}\right), i=1, \ldots, n$,

$$
\phi_{x, y_{1}, \ldots, y_{n}} \in \operatorname{Mor}(\mathcal{B}): \operatorname{Obj}(C)^{\bigotimes \sum k_{i}} \rightarrow \operatorname{Mor}(C)
$$

with a commutative diagram

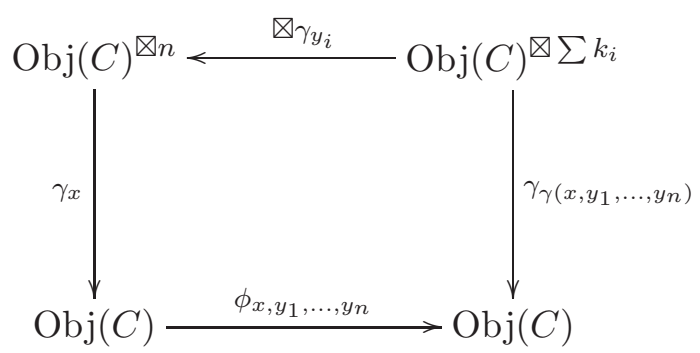

and corresponding coherence diagrams, mimicking the coherence diagrams in the case of sets. One also must not forget to include coherence isomorphisms corresponding to permutations (the $\Sigma_{n}$-action on the $n$th space of an operad), and coherence diagrams corresponding to axioms involving composition and equivariance (cf. [May72]). The fact that 'the targets of $\phi_{x, y_{1}, \ldots, y_{n}}$ are iso' is expressed, for example, by giving an 'inverse' map

$$
\psi_{x, y_{1}, \ldots, y_{n}} \in \operatorname{Mor}(\mathcal{B}): \operatorname{Obj}(C)^{\otimes \sum k_{i}} \rightarrow \operatorname{Mor}(C) .
$$

Thus, we get the following.

Proposition 8. Let $\Gamma$ be a lax $\mathcal{C}$-algebra enhanced over $\mathcal{B}$. Then $B \Gamma$ is canonically a $\overline{\mathcal{C}}$-algebra in $\mathcal{B}$.

\section{Lax algebras enhanced over categories}

We shall now need to consider an even further generalization. Let us, again, first work in the context of simplicial sets, where the structure is simpler than in the $\mathcal{B}$-enhanced case. In this case, the appropriate notion is a lax $\mathcal{C}$-algebra $\Gamma$ enhanced over categories. This means that $\Gamma$ has a structure analogous to that of lax $\mathcal{C}$-algebra, where both $\operatorname{Obj}(\Gamma), \operatorname{Mor}(\Gamma)$ are categories (which we will refer to as the vertical categories), and all structure maps of lax $\mathcal{C}$-algebra are functors (and coherence diagrams commute strictly, rather than just up to natural isomorphisms). Therefore, in addition to the vertical categories, we get two lax $\mathcal{C}$-algebras

$$
\left(\operatorname{Obj}_{\mathrm{Obj}(\Gamma)}, \operatorname{Obj}_{\operatorname{Mor}(\Gamma)}\right)
$$

and

$$
\left(\operatorname{Mor}_{\mathrm{Obj}(\Gamma)}, \operatorname{Mor}_{\mathrm{Mor}(\Gamma)}\right) \text {. }
$$

(We use the subscript notation to distinguish this structure from the vertical categories.) In particular, then, (24), (25) are categories, and we will refer to them as the horizontal categories. To spell 


\section{P. Hu, I. Kriz And A. A. Voronov}

out our notation completely, the vertical categories then are

$$
\begin{aligned}
& \operatorname{Obj}(\Gamma)=\left(\operatorname{Obj}_{\operatorname{Obj}(\Gamma)}, \operatorname{Mor}_{\operatorname{Obj}(\Gamma)}\right), \\
& \operatorname{Mor}(\Gamma)=\left(\operatorname{Obj}_{\operatorname{Mor}(\Gamma)}, \operatorname{Mor}_{\operatorname{Mor}(\Gamma)}\right) .
\end{aligned}
$$

Now suppose we are given a lax $\mathcal{C}$-algebra $\Gamma$ enhanced over categories. Then performing the horizontal bar construction (which we will denote by $B_{\text {hor }}$ ), we obtain a strict simplicial $\overline{\mathcal{C}}$-algebra enhanced over categories (using the vertical structure):

$$
B_{\text {hor }}(\Gamma)=\left(\begin{array}{c}
B_{\text {hor }}\left(\operatorname{Obj}_{\Gamma}\right) \\
B_{\text {hor }}\left(\operatorname{Mor}_{\Gamma}\right)
\end{array}\right)
$$

Observe that $B_{\text {hor }}(\Gamma)$ is 'almost' a $\mathcal{C} \square \mathcal{C}_{1}$-algebra: the $\mathcal{C}$-algebra structure was just described, and the ' $\mathcal{C}_{1}$-structure' can be pulled back from the (associative) categorical composition. The difficulty with that is that the source of the categorical composition is not the product

$$
B_{\text {hor }}\left(\operatorname{Mor}_{\Gamma}\right) \times B_{\text {hor }}\left(\operatorname{Mor}_{\Gamma}\right) \text {, }
$$

but the fibered product

$$
B_{\text {hor }}\left(\operatorname{Mor}_{\Gamma}\right) \times_{B_{\text {hor }}\left(\mathrm{Obj}_{\Gamma}\right)} B_{\text {hor }}\left(\operatorname{Mor}_{\Gamma}\right) .
$$

There is a natural inclusion of (27) to (26). We need a technique for extending the domain of the composition product from (27) to (26).

The technique we shall use is the two-sided bar construction of monads [May72]. The ground category is the category $\mathcal{G}$ of graphs of $\overline{\mathcal{C}}$-algebras over $B=B_{\text {hor }}\left(\mathrm{Obj}_{\Gamma}\right)$, which means $\overline{\mathcal{C}}$-algebras $X$ with two maps of $\overline{\mathcal{C}}$-algebras

$$
S, T: X \rightarrow B
$$

Then the monad in $\mathcal{G}$ which defines categories in $\overline{\mathcal{C}}$ with objects $B$ (where, as above, composition commutes with $\overline{\mathcal{C}}$-algebra structure) is

$$
D X=\coprod_{n \geqslant 0} \underbrace{X \times_{B} \cdots \times_{B} X}_{n \text { times }} .
$$

On the other hand, the monad which defines monoids in $\overline{\mathcal{C}}$-algebras (i.e. again, we require that the $\overline{\mathcal{C}}$-algebra structure commutes with composition) is

$$
E X=\coprod_{n \geqslant 0} \underbrace{X \times \cdots \times X}_{n \text { times }}
$$

Clearly, we have a map of monads $D \rightarrow E$, and we can therefore consider the two-sided bar construction of monads

$$
B(E, D, X) \text {. }
$$

Then (the realization of) (30) is a monoid in $\overline{\mathcal{C}}$-algebras, i.e. a $\overline{\mathcal{C}} \square \mathcal{C}_{1}$-algebra by pull-back. Of course, we would like to compare the homotopy type of (30) to the homotopy type of $X$. As usual, we have the comparison map

$$
X \stackrel{\simeq}{\longleftarrow} B(D, D, X) \longrightarrow B(E, D, X) .
$$

When is the second map (31) an equivalence? An obvious condition is the following.

Condition A. The object $B=B_{\text {hor }}\left(\mathrm{Obj}_{\Gamma}\right)$ is contractible.

However, Condition A per se unfortunately does not suffice, as we need some local condition. In order to formulate the condition, we must slightly change our context: we shall actually assume that (24), (25) are simplicial categories where the simplicial structure of (24) is constant (we will see in the next section that such situation arises naturally). We may of course always realize to 


\section{Kontsevich's CONJECTURE}

make objects simplicial, but for the purposes of the following condition, $X, B$ are then bisimplicial sets, where in one of the simplicial directions $B$ is constant. The condition reads as follows.

Condition B. Considering the projection map $S \times T: X \rightarrow B \times B$, then for every $b \in(B \times B)_{n}$, and every face $d_{i}$ (respectively, $s_{i}$ ), the face (degeneracy) $(S \times T)^{-1} b \rightarrow(S \times T)^{-1} d_{i} b,(S \times T)^{-1} b \rightarrow$ $(S \times T)^{-1} s_{i} b$ are equivalences.

Lemma 9. Assuming Condition B, every projection map

$$
\underbrace{X \times{ }_{B} \cdots \times{ }_{B} X}_{n \text { times }} \rightarrow B^{\times(n+1)}
$$

is a quasifibration.

Proof. It suffices to prove that $S \times T: X \rightarrow B \times B$ is a quasifibration. The Bousfield-Friedlander theorem [BF78, Theorem B.4] implies that such map is a quasifibration provided that, in their terminology:

(i) the vertical homotopy groups $\pi_{n}^{v}(X, x)$ satisfy the $\pi_{*}$-Kan condition for all $n \geqslant 1$;

(ii) the simplicial map $p: \pi_{0}^{v}(X) \rightarrow B \times B$ is a Kan fibration.

However, these assumptions follow from our Condition B.

So, if $F$ is any fiber of (32), we have a diagram

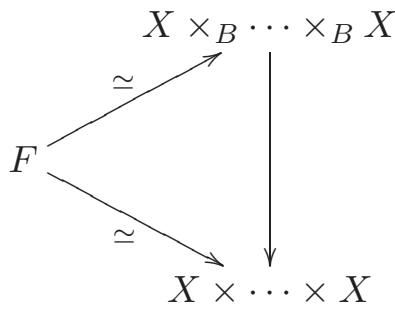

and hence the vertical map is an equivalence. It follows that the right-hand map (31) is an equivalence. Note also that this implies that $X$ is equivalent, via inclusion, to any fiber of any of the maps $S, T$.

Now we need to translate Condition B to some condition on categories which could be applied in the case when $X=B_{\text {hor }}\left(\operatorname{Mor}_{\Gamma}\right)$. The following condition is obviously sufficient $\left(\right.$ Hom $_{\text {vert }}$ denotes vertical Hom sets).

Condition C. Let $M, N, M^{\prime}, N^{\prime} \in \operatorname{Obj}_{\mathrm{Obj}(\Gamma)}$, let $f: M \rightarrow M^{\prime}, g: N \rightarrow N^{\prime}$ be horizontal morphisms. Then the natural projections $S: \operatorname{Hom}_{\text {vert }}(f, g) \rightarrow \operatorname{Hom}_{\text {vert }}(M, N), T: \operatorname{Hom}_{\text {vert }}(f, g) \rightarrow$ $\operatorname{Hom}_{\text {vert }}\left(M^{\prime}, N^{\prime}\right)$ are equivalences, and $S$ is a fibration.

We shall call $\Gamma$ distinguished if Conditions $\mathrm{A}$ and $\mathrm{C}$ are satisfied. We therefore have proven the following.

Proposition 10. Let $\Gamma$ be a distinguished lax $\mathcal{C}$-algebra enhanced over categories. Then for each $M, N \in \operatorname{Obj}_{\mathrm{Obj}(\Gamma)}, \operatorname{Hom}_{\text {vert }}(M, N)$ are naturally equivalent to each other and, moreover, naturally equivalent to a $\overline{\mathcal{C}} \square \mathcal{C}_{1}$-algebra.

Remark. Recall that May's two-sided bar construction of monads [May72] allows us, for any map of operads in simplicial sets $\mathcal{D}_{1} \rightarrow \mathcal{D}_{2}$ which is an equivalence and such that for all $n$ the action of $\Sigma_{n}$ on $\mathcal{D}_{i}(n)$ is free and for any $\mathcal{D}_{1}$-algebra $X$, to construct the equivalent $\mathcal{D}_{2}$-algebra

$$
B\left(D_{2}, D_{1}, X\right)
$$




\section{P. Hu, I. Kriz And A. A. Voronov}

where $D_{i}$ are the monads associated with the operads $\mathcal{D}_{i}$. Therefore, if in Proposition $10, \mathcal{C} \square \mathcal{C}_{1}(n)$ is $\Sigma_{n}$-free, so is $\overline{\mathcal{C}} \square \mathcal{C}_{1}(n)$ (since it maps into $\mathcal{C} \square \mathcal{C}_{1}(n)$ ), and we are allowed to replace $\overline{\mathcal{C}} \square \mathcal{C}_{1}$ with $\mathcal{C} \square \mathcal{C}_{1}$.

Now to treat the case enhanced over a symmetric monoidal category $\mathcal{B}$, we start by defining a category $\Gamma$ enhanced in $\mathcal{B}$-categories. Such structure consists of the following data. First, we have a 'horizontal object category'

$$
\mathrm{Obj}_{\Gamma}=\left(\mathrm{Obj}_{\mathrm{Obj}(\Gamma)}, \mathrm{Obj}_{\mathrm{Mor}(\Gamma)}\right)
$$

which is an ordinary category (i.e. 'enhanced' only over Sets).

Next, we have a 'vertical object category' given by specifying, for each $x, y \in \operatorname{Obj}_{\mathrm{Obj}(\Gamma)}$, a

$$
\operatorname{Hom}(x, y) \in \operatorname{Obj}(\mathcal{B}) \text {. }
$$

Further, there are specified maps

$$
\begin{gathered}
I d \in \operatorname{Mor}(\mathcal{B}): 1_{\square} \rightarrow \operatorname{Hom}(x, x) \\
\gamma \in \operatorname{Mor}(\mathcal{B}): \operatorname{Hom}(x, y) \otimes \operatorname{Hom}(y, z) \rightarrow \operatorname{Hom}(x, z)
\end{gathered}
$$

with usual axioms of associativity and unity and a 'vertical morphism category' specifying, similarly, for $f, g \in \mathrm{Obj}_{\operatorname{Mor}(\Gamma)}$, a

$$
\operatorname{Hom}(f, g) \in \operatorname{Obj}(\mathcal{B})
$$

Further, there are specified maps

$$
\begin{gathered}
I d \in \operatorname{Mor}(\mathcal{B}): 1_{\square} \rightarrow \operatorname{Hom}(f, f) \\
\gamma \in \operatorname{Mor}(\mathcal{B}): \operatorname{Hom}(f, g) \otimes \operatorname{Hom}(g, h) \rightarrow \operatorname{Hom}(f, h)
\end{gathered}
$$

with usual axioms of associativity and unity. (Note, we see that the vertical category is, in fact, enriched over $\mathcal{B}$ in the usual sense - see the remark in the previous section.)

Next, we have a 'horizontal morphism category' $\operatorname{Mor}_{\Gamma}$ enhanced over $\mathcal{B}$ : For

$$
\begin{aligned}
& x_{0} \stackrel{f_{1}}{\longrightarrow} x_{1} \stackrel{f_{2}}{\longrightarrow} x_{2} \\
& y_{0} \stackrel{g_{1}}{\longrightarrow} y_{1} \stackrel{g_{2}}{\longrightarrow} y_{2}
\end{aligned}
$$

in $\operatorname{Obj}_{\Gamma}$, morphisms $T \in \operatorname{Mor}(\mathcal{B}): \operatorname{Hom}\left(f_{1}, g_{1}\right) \rightarrow \operatorname{Hom}\left(x_{1}, y_{1}\right), S \in \operatorname{Mor}(\mathcal{B}): \operatorname{Hom}\left(f_{2}, g_{2}\right) \rightarrow$ $\operatorname{Hom}\left(x_{1}, y_{1}\right)$ and

$$
\gamma \in \operatorname{Mor}(\mathcal{B}): \operatorname{Hom}\left(f_{1}, g_{1}\right) \prod_{\operatorname{Hom}\left(x_{1}, y_{1}\right)} \operatorname{Hom}\left(f_{2}, g_{2}\right) \rightarrow \operatorname{Hom}\left(f_{2} f_{1}, g_{2} g_{1}\right)
$$

and also

$$
I d \in \operatorname{Mor}(\mathcal{B}): \operatorname{Hom}(x, x) \rightarrow \operatorname{Hom}\left(I d_{x}, I d_{x}\right) .
$$

Finally, there is a diagram of commutativity between vertical and horizontal composition. This diagram expresses the equality of two maps from

$$
\left(\operatorname{Hom}\left(f_{1}, g_{1}\right) \prod_{\operatorname{Hom}\left(x_{1}, y_{1}\right)} \operatorname{Hom}\left(f_{2}, g_{2}\right)\right) \otimes\left(\operatorname{Hom}\left(g_{1}, h_{1}\right) \prod_{\operatorname{Hom}\left(y_{1}, z_{1}\right)} \operatorname{Hom}\left(g_{2}, h_{2}\right)\right)
$$

to

$$
\operatorname{Hom}\left(f_{2} f_{1}, h_{2} h_{1}\right)
$$

The first map maps (33) to

$$
\operatorname{Hom}\left(f_{2} f_{1}, g_{2} g_{1}\right) \otimes \operatorname{Hom}\left(g_{2} g_{1}, h_{2} h_{1}\right)
$$




\section{KOntSEviCH's CONJECTURE}

by horizontal composition, and then maps to (34) by vertical composition. The second map maps (33) to

$$
\left(\operatorname{Hom}\left(f_{1}, g_{1}\right) \otimes \operatorname{Hom}\left(g_{1}, h_{1}\right)\right) \prod_{\left(\operatorname{Hom}\left(x_{1}, y_{1}\right) \otimes \operatorname{Hom}\left(y_{1}, z_{1}\right)\right)}\left(\operatorname{Hom}\left(f_{2}, g_{2}\right) \otimes \operatorname{Hom}\left(g_{2}, h_{2}\right)\right)
$$

using the limit properties of a pullback, followed by a map to

$$
\operatorname{Hom}\left(f_{1}, h_{1}\right) \prod_{\operatorname{Hom}\left(x_{1}, z_{1}\right)} \operatorname{Hom}\left(f_{2}, h_{2}\right)
$$

by vertical composition, and then to (34) by horizontal composition.

Now the axioms of a lax $\mathcal{C}$-algebra $\Gamma$ enhanced in $\mathcal{B}$-categories consists of the following data.

(1) An ordinary lax $\mathcal{C}$-algebra structure on $\mathrm{Obj}_{\Gamma}$.

(2) A structure of lax $\mathcal{C}$-algebra enhanced in $\mathcal{B}$ on $\operatorname{Mor}_{\Gamma}$, compatible with the fibering of $\operatorname{Mor}_{\Gamma}$ over $\mathrm{Obj}_{\Gamma}$.

(3) Compatibility diagrams of (2) with vertical unit and composition.

Similarly as before, we can also make $\Gamma$ a simplicial object in the kind of structures just described, and for the homotopical part of our discussion we will find it advantageous to also assume that, in addition to the horizontal category, $\mathrm{Obj}_{\Gamma}$ is simplicially constant.

Now to obtain an analogue of Proposition 10 for lax algebras enhanced over $\mathcal{B}$-categories, we will examine the construction leading up to Proposition 10, noting along the way how they must be changed in view of $\mathcal{B}$-enrichment.

First, we examine $B_{\text {hor }}(\Gamma)$. We see that $B_{\text {hor }}\left(\operatorname{Obj}_{\Gamma}\right)$ is a $\overline{\mathcal{C}}$-algebra over sets, and $B_{\text {hor }}\left(\operatorname{Mor}_{\Gamma}\right)$ is a $\overline{\mathcal{C}}$-algebra enhanced over $\mathcal{B}$ fibered over $B=B_{\text {hor }}\left(\operatorname{Obj}_{\Gamma}\right)$. We further have associative composition, which is a map of $\overline{\mathcal{C}}$-algebras,

$$
B_{\text {hor }}\left(\operatorname{Mor}_{\Gamma}\right) \bigotimes_{B} B_{\text {hor }}\left(\operatorname{Mor}_{\Gamma}\right) \rightarrow B_{\text {hor }}\left(\operatorname{Mor}_{\Gamma}\right)
$$

(the symbol $\otimes_{B}$ indicates $\otimes$ applied fiber-wise). Again, what we want is to be able to extend the product in a way so that we can replace $\otimes_{B}$ with $\nabla$. Again, we have a category of $\mathcal{B}$-enhanced $\overline{\mathcal{C}}$-algebras fibered over $B$, and a map of monads $D \rightarrow E$ in this category,

$$
\begin{gathered}
D X=\coprod_{n \geqslant 0} \underbrace{X \otimes_{B} \cdots \otimes_{B} X}_{n \text { times }}, \\
E X=\coprod_{n \geqslant 0} \underbrace{X \otimes \cdots \otimes X}_{n \text { times }},
\end{gathered}
$$

may then form the two-sided bar construction of monads (30) and note that (30) is a $\mathcal{B}$-enhanced $\overline{\mathcal{C}} \square \mathcal{C}_{1}$-algebra. Therefore, we must again find conditions when the second map (31) is an equivalence, when $X=B_{\text {hor }}\left(\mathrm{Mor}_{\Gamma}\right)$. The conditions we arrive at are again Condition $\mathrm{A}$ and Condition C, although while Condition A does not change, in Condition $\mathrm{C}$ the $\mathrm{Hom}_{\text {vert }}$ sets now denote objects of $s \mathcal{B}$. When these conditions are satisfied, we say, again, that $\Gamma$ is distinguished. We therefore have an enhanced analogue of Proposition 10.

Proposition 11. Let $\Gamma$ be a distinguished lax $\mathcal{C}$-algebra enhanced over $\mathcal{B}$-categories. Then for $M, N \in \operatorname{Obj}_{\operatorname{Obj}(\Gamma)}$, $\operatorname{Hom}_{\mathrm{vert}}(M, N)$ are all naturally equivalent, and naturally equivalent to a $\overline{\mathcal{C}} \square \mathcal{C}_{1}$-algebra enhanced over $\mathcal{B}$. 


\section{P. Hu, I. KRIZ And A. A. Voronov}

\section{Proof of Theorem 3}

In view of Proposition 11, it suffices to produce a distinguished lax $\mathcal{C}$-algebra $\Gamma$ enhanced in $\mathcal{B}$-categories, where for $M, N \in \operatorname{Obj}_{\mathrm{Obj}(\Gamma)}$,

$$
\operatorname{Hom}_{\text {vert }}(M, N)
$$

is naturally equivalent to $(11)$.

Let $Q$ be a cofibrant fibrant special operad in $s \mathcal{B}$ fibered over $\mathcal{C}$, a cofibrant operad in simplicial sets with $\mathcal{C}(0)=*, \mathcal{C}(1) \simeq *$. Define the category $\Gamma$ enhanced in $s \mathcal{B}$-categories as follows: if $\mathcal{B}=$ $K$-modules, the horizontal category $\mathrm{Obj}_{\Gamma}$ has as objects all fibration equivalences of $Q_{1}$-modules

$$
M \rightarrow Q(0)
$$

where $M$ is cofibrant in the category of $Q_{1}$-modules (note that $Q(0)$ is fibrant). If $\mathcal{B}=$ Sets, drop the requirement that (35) be a fibration. The (horizontal) morphisms in $\mathrm{Obj}_{\Gamma}$ are commutative diagrams

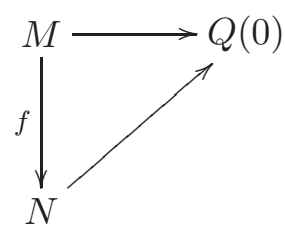

where the map $f: M \rightarrow N$ is a cofibration in the category of $Q_{1}$-modules. The vertical Hom-sets in $\operatorname{Obj}(\Gamma)$ (i.e. between two objects of the form (35)) are

$$
\operatorname{Hom}_{Q_{1}}\left(M, M^{\prime}\right),
$$

if $\mathcal{B}=K$-modules

When $\mathcal{B}=$ Sets, we could actually use the space

$$
\operatorname{Map}_{\left|Q_{1}\right|}\left(|M|,\left|M^{\prime}\right|\right) \text {. }
$$

However, since we want to stay in the category of simplicial sets, we get back by applying the singular set functor to (37).

The vertical Hom-sets in $\operatorname{Mor}(\Gamma)$ are groups of pairs (under addition) of

$$
(u, v) \in \operatorname{Hom}_{Q_{1}}(M, N) \prod \operatorname{Hom}_{Q_{1}}\left(M^{\prime}, N^{\prime}\right)
$$

for $K$-modules and

$$
(u, v) \in \operatorname{Map}_{\left|Q_{1}\right|}(|M|,|N|) \prod \operatorname{Map}_{\left|Q_{1}\right|}\left(\left|M^{\prime}\right|,\left|N^{\prime}\right|\right)
$$

for sets which make the following diagram commutative.

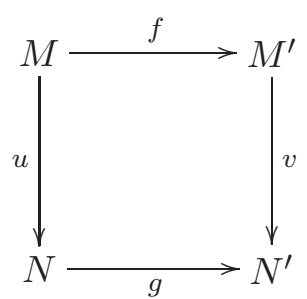

Note that the requirement that $f$ be a cofibration implies (for $K$-modules) that the map induced by $f$

$$
\operatorname{Hom}\left(M^{\prime}, N^{\prime}\right) \rightarrow \operatorname{Hom}\left(M, N^{\prime}\right)
$$




\section{KONTSEVICH's CONJECTURE}

is a fibration equivalence. Therefore,

$$
\begin{gathered}
\operatorname{Hom}(f, g)=\operatorname{Hom}(M, N) \prod_{\operatorname{Hom}\left(M, N^{\prime}\right)} \operatorname{Hom}\left(M^{\prime}, N^{\prime}\right) \\
\simeq \operatorname{Hom}(M, N)
\end{gathered}
$$

is a fibration equivalence. Now we have the following diagram (where the arrows are induced maps).

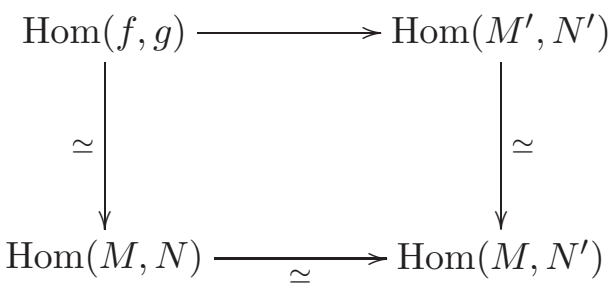

The bottom row is an equivalence because $N \rightarrow N^{\prime}$ is an equivalence, and by our hypothesis, $N, N^{\prime}$ are fibrant. Similarly for sets. This already proves condition Condition C.

Now $Q$ being special implies that $\Gamma$ is a lax $\mathcal{C}$-algebra enhanced over $s \mathcal{B}$-categories. In effect, the lax $\mathcal{C}$-algebra structure is defined as follows: for $x \in \mathcal{C}(n)$, and $Q_{1}$-modules $M_{1}, \ldots, M_{n}$, the $x$-product of $M_{1}, \ldots, M_{n}$ is

$$
Q(n)_{x} \bigotimes_{Q_{1}^{\bigotimes_{n}}}\left(M_{1} \otimes \cdots \otimes M_{n}\right) .
$$

If $Q$ is special, cofibrant and fibrant, the canonical map from (38) to $Q(0)$ is an equivalence (see (7)). On morphisms, (38) preserves $Q_{1}$-cofibrations if $Q$ is cofibrant. For $\mathcal{B}=K$-modules, (38) preserves fibrations, because fibrations of simplicial $K$-modules are precisely onto maps. Note that this operation is functorial, and on morphisms carries cofibrations to cofibrations, with $s \mathcal{B}$ as above.

Thus, the proof of the statement that $\Gamma$ is distinguished (and, hence, of Theorem 3 ) is reduced to the following.

Proposition 12. We have

$$
B \mathrm{Obj}_{\Gamma} \simeq *
$$

Proof. To show (39), one chooses a particular object

$$
M_{0} \rightarrow Q(0)
$$

For any object of $\mathrm{Obj}_{\Gamma}$

$$
M \rightarrow Q(0)
$$

one can obviously choose an arrow $N_{M} \rightarrow Q(0)$ in $\mathrm{Obj}_{\Gamma}$ together with the following diagram in $\mathrm{Obj}_{\Gamma}$.

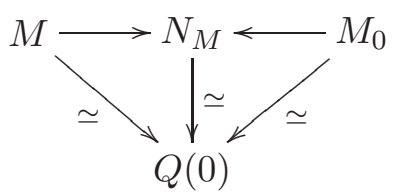

Similarly, for a (horizontal) morphism in $\mathrm{Obj}_{\Gamma}$

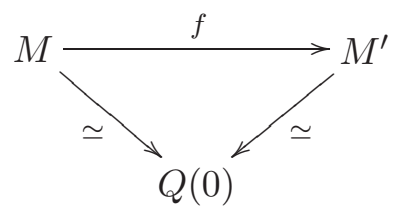




\section{P. Hu, I. Kriz And A. A. Voronov}

there is an $N_{f} \rightarrow Q(0)$ in $\mathrm{Obj}_{\Gamma}$ such that, over $Q(0)$, we have the following commutative diagram.

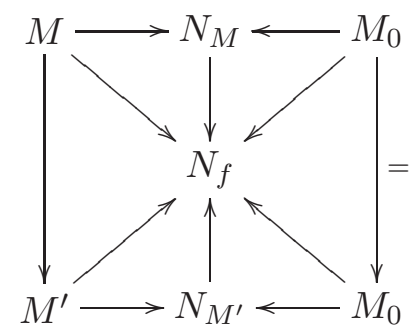

To formalize the procedure this will give, recall the barycentric subdivision $C^{\prime}$ of a category $C$ (in our case, $C=\mathrm{Obj}_{\Gamma}$ ): the category $C^{\prime}$ is a partially ordered set whose objects are $n$-tuples of composable arrows in $C$

$$
x_{0} \stackrel{f_{1}}{\longrightarrow} x_{1} \longrightarrow \cdots \stackrel{f_{n}}{\longrightarrow} x_{n},
$$

and a $k$-tuple of composable morphisms

$$
\stackrel{g_{1}}{\longrightarrow} \cdot \longrightarrow \stackrel{g_{k}}{\longrightarrow} \cdot
$$

is said to be less than or equal to (40) if the $g_{i}$ are obtained by consecutive compositions (or omissions from the beginning or end) of the $f_{i}$. Then there is a canonical functor

$$
\Psi: C^{\prime} \rightarrow C
$$

which on objects is given by

$$
\left(f_{n}, \ldots, f_{1}\right) \mapsto T f_{n}
$$

(and by the obvious formula on morphisms). The functor $\Psi$ induces an equivalence upon applying $B$ (the bar construction).

Now repeating the procedure we described constructs a functor

$$
N:\left(\mathrm{Obj}_{\Gamma}\right)^{\prime} \rightarrow \mathrm{Obj}_{\Gamma},
$$

together with natural transformations

$$
\begin{aligned}
& \Psi \rightarrow N \\
& G \rightarrow N
\end{aligned}
$$

where $G$ is the constant functor with value in $M_{0} \rightarrow Q(0)$, thus showing that $B \mathrm{Obj}_{\Gamma}$ is contractible, as claimed.

\section{The special property for $k$-cubes}

The purpose of this section is to prove Theorem 6 . In this section $\mathcal{C}_{k}$ will stand for the topological version of the little $k$-cube operad, and we will also consider the topological version of the construction (12), $\mathcal{C}=\mathcal{C}_{k}$. Recall that $D_{0}(X)=C_{k} X$. It clearly suffices to prove that the map

$$
\phi: B\left(D_{n} X,\left(D_{1} X\right)^{\times n},\left(D_{0} X\right)^{\times n}\right) \rightarrow C_{k} X \times B\left(\mathcal{C}_{k}(n), \mathcal{C}_{k}(1)^{\times n}, \mathcal{C}_{k}(0)^{\times n}\right)
$$

is an equivalence, where the first coordinate of the map $\phi$ is given by composition, and the second map by the forgetful map

$$
D_{\ell}(X) \rightarrow \mathcal{C}_{k}(\ell)
$$

In effect, to get from spaces to simplicial sets, we may apply the singular set functor, and to get to $K$-modules, we may further apply the free $K$-module functor.

Now since the left-hand side of (43) obviously preserves weak equivalences, we can further replace the terms of $(43)$ as follows. First, let $\mathcal{C}_{k}^{\prime}(\ell) \subset \mathcal{C}_{k}(\ell)$ consist of all $\ell$-tuples of little cubes $\alpha_{1}, \ldots, \alpha_{\ell}: I^{k} \rightarrow I^{k}$ such that $\operatorname{Im}\left(\alpha_{i}\right) \subset \operatorname{Int}\left(I^{k}\right)$ and the images $\operatorname{Im}\left(\alpha_{i}\right)$ are disjoint. Now let $M(\ell)$ 


\section{KOntSEviCH's CONJECTURE}

be the space of pairs $(\alpha, m)$ where $\alpha=\left(\alpha_{1}, \ldots, \alpha_{\ell}\right) \in \mathcal{C}_{k}(\ell)$ and $m$ is a set of unordered $X$-decorated points in $I^{k}-\bigcup_{i=1}^{\ell} \operatorname{Im}\left(\alpha_{i}\right)$ (with the usual configuration space topology). Let $M^{\prime}(\ell)$ be defined in the same way as $M(\ell)$, with $\mathcal{C}_{k}(\ell)$ replaced by $\mathcal{C}_{k}^{\prime}(\ell)$ :

$$
M^{\prime}(\ell)=M(\ell) \times_{\mathcal{C}_{k}(\ell)} \mathcal{C}_{k}^{\prime}(\ell) .
$$

Then we have equivalences

$$
D_{n}(X) \rightarrow M(n) \leftarrow M^{\prime}(n)
$$

(the first map replaces a little cube decorated by an element of $X$ by its center). Thus, we can further restate the claim that (43) is an equivalence as follows.

Proposition 13. The map

$$
\kappa: B\left(M^{\prime}(\ell), M(1)^{\times \ell}, M(0)^{\times \ell}\right) \rightarrow M(0) \times B\left(\mathcal{C}_{k}^{\prime}(\ell), \mathcal{C}_{k}(1)^{\times \ell}, \mathcal{C}_{k}(0)^{\times \ell}\right)
$$

where the first map is by composition and the second map is by projection $M \rightarrow \mathcal{C}$, is an equivalence.

Remark. Recall that $\mathcal{C}_{k}^{\prime}(\ell) \simeq \mathcal{C}_{k}(\ell), \mathcal{C}_{k}(1) \simeq *$, so the right-hand side of (44) is weakly equivalent to $M(0) \times \mathcal{C}_{k}(\ell)$.

Proof of Proposition 13. First define $N(\ell)$ as the pull-back

$$
N(\ell)=\left(\mathcal{C}_{k}^{\prime}(\ell) \times \mathcal{C}_{k}(1)^{\ell}\right) \times_{\mathcal{C}_{k}^{\prime}(\ell)} M^{\prime}(\ell)
$$

where the map

$$
\mathcal{C}_{k}^{\prime}(\ell) \times \mathcal{C}_{k}(1)^{\ell} \rightarrow \mathcal{C}_{k}^{\prime}(\ell)
$$

is by composition. Then $N(\ell)$ enjoys a right $M(1)^{\ell}$-action where $M(1)^{\ell}$ acts by composition on $M^{\prime}(\ell)$ and by the forgetful map together with internal composition on $\mathcal{C}_{k}^{\prime}(\ell) \times \mathcal{C}_{k}(1)^{\ell}$ :

$$
\begin{aligned}
\left(\mathcal{C}_{k}^{\prime}(\ell) \times \mathcal{C}_{k}(1)^{\ell}\right) \times M(1)^{\ell} \longrightarrow \mathcal{C}_{k}^{\prime}(\ell) \times \mathcal{C}_{k}(1)^{\ell} \times \mathcal{C}_{k}(1)^{\ell} & \\
& \mid I d \times \gamma^{\ell} \\
\mathcal{C}_{k}^{\prime}(\ell) & \times \mathcal{C}_{k}(1)^{\ell} .
\end{aligned}
$$

Furthermore, the projection

$$
N(\ell) \rightarrow M^{\prime}(\ell)
$$

is obviously an equivalence (by contracting $\left.\mathcal{C}_{k}(1)^{\ell}\right)$, so we may replace $M^{\prime}(\ell)$ by $N(\ell)$ in the statement of the proposition.

Now filter $N(\ell)$ by closed subspaces $N_{q}(\ell)$ consisting of all triples

$$
(\alpha, \beta, x),
$$

$\alpha=\left(\alpha_{1}, \ldots, \alpha_{\ell}\right) \in \mathcal{C}_{k}^{\prime}(\ell), \beta=\left(\beta_{1}, \ldots, \beta_{\ell}\right) \in \mathcal{C}_{k}^{\prime}(1)^{\ell}, x \in M^{\prime}(\ell)$ where the number of $X$-decorated points of $x$ contained in

$$
I^{k}-\bigcup_{i=1}^{\ell} \operatorname{Im}\left(\alpha_{i}\right)
$$

is less than or equal to $q$.

Lemma 14. Suppose $A \subset X$ is an $M$-equivariant weak neighborhood deformation retract (NDR) pair with Urysohn function and homotopy $(u, h)$ where $M$ is a monoid acting on the right, such that the following conditions are satisfied.

(1) $X-A=V \times M$ for some $V \subset X-A$ closed.

(2) $h_{t}(A \cup V) \subset A \cup V$ for all $t$. (Note that $A \cup V=A \cup C l(V)$; $C l$ denotes closure.) 


\section{P. Hu, I. KRIZ And A. A. Voronov}

Then there is a natural $M$-equivariant homotopy equivalence

$$
\text { Cofiber }(A \subset X) \rightarrow((A \cup V) / A) \wedge M_{+} .
$$

Proof. Because of the weak NDR property, we have

$$
\text { Cofiber }(A \subset X) \simeq X / A,
$$

so it suffices to prove

$$
X / A \simeq(A \cup V) / A \wedge M_{+} .
$$

To get a map $\leftarrow$, note that the inclusion

$$
A \cup V \rightarrow X
$$

extends to an equivariant map

$$
(A \cup V) \times M \rightarrow X \rightarrow X / A
$$

which clearly annihilates $A \times M$, thus inducing

$$
\psi:(A \cup V) \times M / A \times M \rightarrow X / A .
$$

To get a map $\rightarrow$ in (47), use the map $\phi$ induced by $h_{1}$ : to see that it is continuous, let

$$
U=\{x \in X \mid u(x)<1\} .
$$

Then $\phi$ is constant (hence, continuous) on $U$, but also continuous on $X-A$. Now $\{U, X-A\}$ is an open cover of $X$.

Now since $\psi$ is obviously a bijection, we can define both homotopies $\phi \psi \simeq I d, \psi \phi \simeq I d$ as $h_{t}$. Then $\psi \phi$ is a quotient of $h_{t}$ (with topology), and thus is continuous. To see that

$$
h_{t}: \phi \psi \simeq I d
$$

is continuous, note that we have a continuous map

$$
h_{t}: A \cup V \rightarrow A \cup V,
$$

and hence

$$
\text { unit } \circ h_{t}: A \cup V \rightarrow(A \cup V) \wedge M_{+} .
$$

Then (48) is the free extension of (49).

Now we shall prove the following.

Lemma 15. The pair $N_{q-1}(\ell) \subset N_{q}(\ell)$, with $M=M(1)^{\ell}$, satisfy the hypotheses of Lemma 14 .

Thus, so do $B\left(N_{q-1}(\ell), M(1)^{\ell}, M(1)^{\ell}\right) \subset B\left(N_{q}(\ell), M(1)^{\ell}, M(1)^{\ell}\right)$.

Now consider the following diagram.

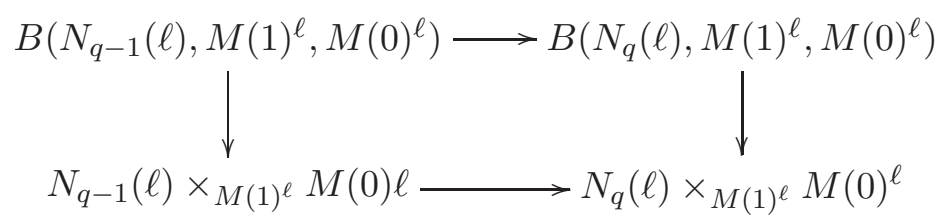

By Lemma 14, the cofibers of both rows are naturally equivalent to

$$
((A \cup V) / A) \wedge M(0)_{+}^{\ell} .
$$

Thus, if the left column of (50) is an equivalence, so is the right column. Thus, inductively,

$$
B\left(N(\ell), M(1)^{\ell}, M(0)^{\ell}\right) \simeq N(\ell) \times_{M(1)^{\ell}} M(0)^{\ell} \cong \mathcal{C}_{k}^{\prime}(\ell) \times M(0) .
$$

The equivalences are easily checked to be compatible with the required maps. 


\section{KOntSEviCH's CONJECTURE}

Proof of Lemma 15. To simplify notation, we shall identify little cubes with their images. For a little cube $\alpha$ in $I^{k}$, and for $t \in \mathbb{R}_{>0}$, let $t \alpha$ be the cube with the same center which is $t$ times larger.

Now first note that there is a continuous function

$$
\lambda: \mathcal{C}_{k}^{\prime}(\ell) \rightarrow \mathbb{R}_{>1}
$$

such that for $\alpha=\left(\alpha_{1}, \ldots, \alpha_{\ell}\right) \in \mathcal{C}_{k}^{\prime}(\ell), \lambda=\lambda(\alpha)$,

$$
\left(\lambda \alpha_{1}, \ldots, \lambda \alpha_{\ell}\right) \in \mathcal{C}_{k}^{\prime}(\ell) .
$$

To this end, for every $\beta \in \mathcal{C}_{k}^{\prime}(\ell)$, there is an open neighborhood $U$ of $\beta$ and a constant $\mu>1$ which works as $\lambda$ in (51) for $\alpha \in U$. Thus, since $\mathcal{C}_{k}^{\prime}(\ell)$ is paracompact, the function $\lambda$ can constructed by partition of unity.

Now also note that for two pairs of cubes $\alpha \supset \beta, \gamma \supset \delta$ (not equal), there is a canonical homeomorphism

$$
\Phi_{\gamma \delta}^{\alpha \beta}: C l(\beta-\alpha) \rightarrow C l(\delta-\gamma) .
$$

First, there are canonical linear homeomorphisms identifying the boundaries $\partial \alpha, \partial \beta, \partial \gamma, \partial \delta$. Connecting two corresponding points on $\partial \alpha, \partial \beta$ creates a line segment; map this segment linearly onto the line segment obtained by identifying the corresponding points on $\partial \gamma$, $\partial \delta$. Also let $\left(\Phi_{\gamma \delta}^{\alpha \beta}\right)_{*}$ be the map induced by $\Phi_{\gamma \delta}^{\alpha \beta}$ on configuration spaces.

Then define the homotopy $h$ in $N_{q}(\ell)$ (see (45)) by

$$
h_{t}(\alpha, 1, m)=\left(\left(\alpha_{i}\right)_{i=1}^{\ell},((1-t / 3))_{i=1}^{\ell},\left(\left(\Phi_{\lambda(\alpha) \alpha_{i},(1-t / 3) \alpha_{i}}^{\lambda(\alpha) \alpha_{i}, \alpha_{i}}\right)_{i=1}^{\ell}\right)_{*} m\right),
$$

and extend $M(1)^{\ell}$-equivariantly. We can let

$$
\text { V }
$$

consist of all triples $(\alpha, 1, m)$ (see $(45))$ where $m$ contains exactly $q X$-decorated points. Define

$$
u(\alpha, \beta, m)=\sup \left\{t \mid \begin{array}{l}
\text { there are exactly } q X \text {-decorated points in } \\
\left(\left(\Phi_{\lambda(\alpha) \alpha_{i},(1-t / 3) \alpha_{i}}^{\lambda(\alpha) \alpha_{i}, \alpha_{i}}\right)_{i=1}^{\ell}\right)_{*} m \text { which are in } I^{k}- \\
\bigcup_{i=1}^{\ell} \alpha_{i} .
\end{array}\right\}
$$

\section{ACKNOWLEDGEMENT}

We are very indebted to T. Fiore for comments.

\section{REFERENCES}

BKP89 R. Blackwell, G. M. Kelly and A. J. Power, Two-dimensional Monad theory, J. Pure Appl. Algebra 59 (1989), 1-27.

BV73 J. Boardman and R. Vogt, Homotopy invariant algebraic structures on topological spaces, Lecture Notes in Mathematics, vol. 347 (Springer, Berlin, 1973).

BF78 A. Bousfield and E. Friedlander, Homotopy theory of $\Gamma$-spaces, spectra and bisimplicial sets, in Geometric Applications of Homotopy Theory II (Evanston, 1977), Lecture Notes in Mathematics, vol. 658 (Springer, Berlin, 1978), 80-130.

DLM98 C. Dong, H. Li and G. Mason, Twisted representations of vertex operator algebras, Math. Ann. 310 (1998), 571-600.

Dun88 G. Dunn, Tensor product of operads and iterated loop spaces, J. Pure Appl. Algebra 50 (1988), 237-258.

DS95 W. G. Dwyer and J. Spalinski, Homotopy theories and model categories, in Handbook of algebraic topology (North-Holland, Amsterdam, 1995), 73-126. 


\section{KONTSEVICH'S CONJECTURE}

EKMM97 A. D. Elmendorf, I. Kriz, M. A. Mandell and J. P. May, Rings, modules, and algebras in stable homotopy theory, with an appendix by M. Cole, Mathematical Surveys and Monographs, vol. 47 (American Mathematical Society, Providence, RI, 1997).

GK94 V. Ginzburg and M. Kapranov, Koszul duality for operads, Duke Math. J. 76 (1994), 203-272.

Hua97 Y. Z. Huang, Two-dimensional conformal geometry and vertex operator algebras, Progress in Mathematics, vol. 148 (Birkhäuser, Basel, 1997).

Kon99 M. Kontsevich, Operads and motives in deformation quantization, Lett. Math. Phys. 48 (1999), $35-72$.

KS99 M. Kontsevich and Y. Soibelman, Deformations of algebras over operads and the Deligne conjecture, in Conference Moshe Flato 1999, vol. I, Math. Phys. Stud. 21, 255-307.

KM95 I. Kriz and J. P. May, Operad, algebras, modules and motives, Astérisque 233 (1995).

Mac71 S. MacLane, Categories for the working mathematician, Graduate Texts in Mathematics, vol. 5 (Springer, Berlin, 1971).

May72 J. P. May, Geometry of iterated loop spaces, Lecture Notes in Mathematics, vol. 271 (Springer, Berlin, 1972).

MS01 J. McClure and J. Smith, A solution of Deligne's Hochschild cohomology conjecture, in Recent progress in homotopy theory, Baltimore, MD, 2000, Contemp. Math., vol. 293 (American Mathematical Society, Providence, RI, 2002), 153-193.

Qui68 D. Quillen, On the (co-)homology of commutative rings, in Applications of categorical algebra, Proc. Sympos. Pure Math. 17 (1968), 65-87.

Qui67 D. Quillen, Homotopical algebra, Lecture Notes in Mathematics, vol. 43 (Springer, Berlin, 1967).

Tam98 D. E. Tamarkin, Another proof of M. Kontsevich's formality theorem, Preprint (1998), math.QA/9803025.

Tam00 D. E. Tamarkin, The deformation complex of a d-algebra is a $(d+1)$-algebra, Preprint $(2000)$, math.QA/0010072.

Tam03 D. E. Tamarkin, Formality of chain operad of little discs, Lett. Math. Phys. 66 (2003), 65-72.

Vor00 A. A. Voronov, Homotopy Gerstenhaber algebras, in Conference Moshe Flato 1999, vol. II, Math. Phys. Stud. 22 (2000), 307-331.

Zhu96 Y. Zhu, Modular invariance of characters of vertex operator algebras, J. Amer. Math. Soc. 9 (1996), 237-302.

$\mathrm{Po} \mathrm{Hu}$

Department of Mathematics, Wayne State University, 656 W. Kirby,

Rm. 1150 Faculty/Administration Building, Detroit, MI 48202, USA

Igor Kriz ikriz@umich.edu

Department of Mathematics, University of Michigan, 2074 E Hall, 525 E University Avenue, Ann Arbor, MI 48109-1109, USA

Alexander A. Voronov

Department of Mathematics, University of Minnesota, 127 Vincent, 206 Church Street SE,

Minneapolis, MN 55455-0487, USA 